\title{
(Adiabatic) phase boundaries in a bistable chain with twist and stretch
}

\author{
Qingze Zhao and Prashant K. Purohit \\ Department of Mechanical Engineering and Applied Mechanics, \\ University of Pennsylvania, Philadelphia, PA 19104, U.S.A.
}

\begin{abstract}
Mass-spring chains with only extensional degrees of freedom have provided insights into the behavior of crystalline solids, including those capable of phase transitions. Here we add rotational degrees of freedom to the masses in a chain and study the dynamics of phase boundaries across which both the twist and stretch can jump. We solve impact and Riemann problems in the chain by numerical integration of the equations of motion and show that the solutions are analogous to those in a phase transforming rod whose stored energy function depends on both twist and stretch. From the dynamics of phase boundaries in the chain we extract a kinetic relation whose form is familiar from earlier studies involving chains with only extensional degrees of freedom. However, for some combinations of parameters characterizing the energy landscape of our springs we find propagating phase boundaries for which the rate of dissipation, as calculated using isothermal expressions for the driving force, is negative. This suggests that we cannot neglect the energy stored in the oscillations of the masses in the interpretation of the dynamics of mass-spring chains. Keeping this in mind we define a local temperature of our chain and show that it jumps across phase boundaries, but not across sonic waves. Hence, impact problems in our mass-spring chains are analogous to those on continuum thermoelastic bars with Mie-Gruneisen type constitutive laws. At the end of the paper we use our chain to shed some light on experiments involving yarns that couple twist and stretch to perform useful work in response to various stimuli.
\end{abstract}

Keywords:

Phase transition, Twist stretch coupling, Atomic simulation, Kinetic relation

\section{Introduction}

Chains of masses and springs (see figure 1) have been used as models to understand the mechanical and thermal behaviours of crystalline solids for a long time $[13,14]$. In most of these studies the potentials characterizing the springs have one well. When the potentials characterizing the springs have multiple wells then the dynamics of mass-spring chains has been shown to be similar to that of one-dimensional bars capable of phase transitions $[1,2,5,7,9,10,4,15,18,26,31,32,33]$. This insight has been utilized 
to extract kinetic relations for moving phase boundaries by comparing solutions of impact and Riemann problems in continuum bars to those in the bistable chains $[22,32]$. The extracted kinetic relation has a simple analytical form that has been recently shown to be a universal near sonic limit [33]. More recently, mass-spring chains have also provided insights into the mechanics of biological macromolecules [6, 7]. The present work is also motivated by biological examples. However, we will go beyond mass-spring chains in which each mass has just a translational degree of freedom - each of our masses will translate and rotate about the line connecting them. The continuum analogue of this mass-spring chain is a rod that can stretch and twist (but not bend). Our goal is to study the dynamics of phase boundaries in such a rod.

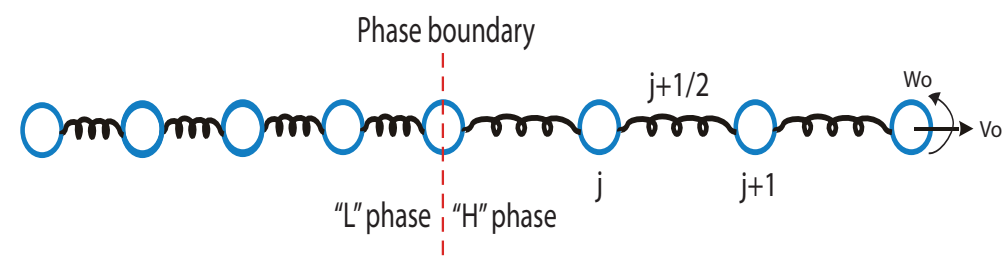

Figure 1: Mass-spring chain in which each mass has an extensional and rotational degree of freedom. The masses are labeled $j, j+1$, etc., and the spring connecting these two masses is labeled $j+\frac{1}{2}$. A phase boundary separates regions of the chain in which springs are in different phases. Springs in the ' $\mathrm{H}$ ' phase correspond to the top right well in the contour plot in figure 2 and the ' $\mathrm{L}$ ' phase correspond to the bottom left well in the contour plot . We specify extensional velocity $v_{0}$ and rotational velocity $w_{0}$ on the masses at the boundary. We can also specify force and moment boundary conditions.

We are inspired by three different biological examples in which twist-stretch phase transitions occur. The first is the dynamics of a primitive motile system regulated by binding of calcium ions in the horseshoe crab sperm [30]. In these organisms a bundle of actin filaments that stores both twisting and stretching energy undergoes a phase transition when calcium binds to the actin filaments. The phase transition is localized and propagates along the length of the bundle releasing energy and causing large conformational changes that result in actuation. Similar phase transitions also occur in DNA which is a rod-like helical polymer capable of stretching, twisting and bending deformations. Bending deformations (caused by external loads or by thermal bombardment) are beyond the scope of this paper but they can be neglected when the tensions are large enough that the DNA is mostly straight and aligned with the direction of the applied tension. Phase transitions can be caused in straight DNA by changing the temperature, applying large forces and twisting moments, or by changing the concentration of ions in the solution [35]. Often, phase transitions in DNA are localized and propagate by the motion of a phase boundary [34]. Our third example also concerns helical filaments forming the tail sheath of a T4 bacteriophage [12]. These filaments undergo a phase transition as soon as the tail binds to a host bacterium causing the radius of the helix to increase and the pitch to decrease, so that the cylinder formed by several of these helices arranged side-by-side inflates (radially) and compresses (axially) to transfer the viral DNA into the host cell. All these examples involve filaments whose lengths are microns or smaller and they are usually surrounded by a viscous fluid, so that inertia forces are 
negligible. We have studied the propagation of phase boundaries in such rods earlier [24,37]. Recently, however, macroscopic yarns (called 'artificial muscles') have been fabricated to perform actuation in which inertia forces cannot be neglected [17]. These filaments are made by twisting and braiding carbon nanotube yarn; they are activated by heat, binding of small molecules, and even light. They can perform useful extensional and rotational work over thousands of cycles. The common features in all of these examples are (a) filaments in which twist and stretch are coupled, and (b) phase transitions driven by ions, forces or heat. The key constitutive information that is needed to study these phase transitions (in dynamic or quasi-static problems) is a kinetic relation. Finding a kinetic relation for twist-stretch phase boundaries is the primary objective of this work.

The paper is laid out as follows. First, we give a short account of the balance laws and constitutive relations for rods capable of phase transitions in twist and stretch. Second, we describe an analogous massspring chain and integrate its dynamics with a leapfrog algorithm. We have simulated both impact and Riemann problems to study the motion of a phase boundary. Third, we extract a kinetic relation from our mass-spring simulations and show that we can correctly solve continuum problems with it. Even though this kinetic relation is useful it must be interpreted with care without neglecting the energy stored in the oscillations of the masses and springs which may represent a local temperature. Lastly, we apply our ideas to some recently developed artificial muscles mentioned above.

\section{Thermomechanics of $1-D$ chain}

\subsection{Balance Laws}

Let us begin by considering phase boundaries propagating in a one-dimensional continuum in the interval $0 \leq x \leq L$. Here $x$ is the reference coordinate along the continuum of length $L$. The specific realization of the continuum we have in our minds is a rod with circular cross-section. At each reference point we have two variables - the deformed position $z(x, t)$ of the rod cross-section located at $x$ at time $t$, and $\alpha(x, t)$ the angle through which that cross-section has rotated at time $t$. We require that $z(x, t)$ and $\alpha(x, t)$ be continuous at all $x$ and $t$ so that the rod does not break. We do not allow bending deformations of the rod. The stretch of the rod is $\lambda(x, t)=\frac{\partial z}{\partial x}$ and the twist is $\kappa(x, t)=\frac{\partial \alpha}{\partial x}$. These quantities are allowed to jump at a finite number of points in our continuum. If one such jump is located at $x=s(t)$ then let us denote $x>s(t)$ as the + side, $x<s(t)$ as the - side. For any quantity $y(x, t)$ we denote $y\left(x_{+}, t\right)-y\left(x_{-}, t\right)$ by $[|y|]$ and $\frac{y\left(x_{+}, t\right)+y(x-, t)}{2}$ by $\langle y\rangle$. From continuity of the deformed material we have $[|z|]=0$ and $[|\alpha|]=0$. Differentiating these two equations with respect to time we get the kinematic jump conditions

$$
\begin{aligned}
& \dot{s}[|\lambda|]+[|\dot{z}|]=0, \\
& \dot{s}[|\kappa|]+[|\dot{\alpha}|]=0 .
\end{aligned}
$$


The equation for balance of linear momentum for a portion of the rod in the interval $\left(x_{1}, x_{2}\right)$ in the absence of body forces is

$$
\frac{d}{d t} \int_{x_{1}}^{x_{2}} \rho \dot{z} d x=\left.T\right|_{x_{1}} ^{x_{2}}
$$

where $\rho(x, t)$ is the mass per unit length and and $T(x, t)$ is the tension in the $\operatorname{rod}[22,24,23]$. If we localize this equation to a discontinuity $s(t)$ (with $x_{1} \leq s(t) \leq x_{2}$ ) then we get the linear momentum jump condition

$$
[|T|]+\dot{s}[|\rho v|]=0,
$$

with $v(x, t)=\dot{z}$. If we localize this away from discontinuity we get

$$
\rho \ddot{z}=\frac{\partial T}{\partial x} .
$$

The equation for the balance of angular momentum for a portion of the rod in the interval $\left(x_{1}, x_{2}\right)$ in the absence of body moments is

$$
\frac{d}{d t} \int_{x_{1}}^{x_{2}} \rho r_{g}^{2} \dot{\alpha} d x=\left.M\right|_{x_{1}} ^{x_{2}},
$$

where $r_{g}$ is the radius of gyration of the cross-section and $M(x, t)$ is the torque in the rod. If we localize this equation to a discontinuity $s(t)$ (with $x_{1} \leq s(t) \leq x_{2}$ ) then we have

$$
[|M|]+\dot{s}\left[\left|\rho r_{g}^{2} w\right|\right]=0
$$

where $w(x, t)=\dot{\alpha}$. If we localize this equation away from the discontinuity at $x=s(t)$ then we get

$$
\rho r_{g}^{2} \ddot{\alpha}=\frac{\partial M}{\partial x} .
$$

Starting from the balance laws given above one can derive an expression for the thermodynamic driving force across a discontinuity. This exercise has been carried out in detail in [21] for the more general case when the 1D continuum can stretch, bend and twist. The expression for the driving force is

$$
f_{\text {driving }}=[|W|]-\langle T\rangle[|\lambda|]-\langle M\rangle[|\kappa|],
$$

where $W(\lambda, \kappa)$ is the Helmholtz free energy per unit length of our continuum.

\subsection{Constitutive law}

We will now choose a specific form for $W(\lambda, \kappa)$. We imagine that $W(\lambda, \kappa)$ has two wells located at different points on the $\lambda-\kappa$ plane. The phase corresponding to low values of $\lambda$ and $\kappa$ is denoted as $j=L$ and the Helmholtz free energy per unit length in this phase is $W_{L}(\lambda, \kappa)$. Similarly, the phase corresponding to large values of $\lambda$ and $\kappa$ is denoted as $j=H$ with free energy density $W_{H}(\lambda, \kappa)$. We assume $W(\lambda, \kappa)$ is a quadratic form in both these phases. Hence,

$$
W_{j}(\lambda, \kappa)=\frac{S_{j}}{2}\left(\lambda-\lambda_{j}^{0}\right)^{2}+g_{j}\left(\lambda-\lambda_{j}^{0}\right)\left(\kappa-\kappa_{j}^{0}\right)+\frac{C_{j}}{2}\left(\kappa-\kappa_{j}^{0}\right)^{2},
$$


where $j$ denotes a phase, $S_{j}$ is the stretching modulus of phase $j, C_{j}$ is the twisting modulus and $g_{j}$ is the twist-stretch coupling modulus. The constants $\lambda_{j}^{0}$ and $\kappa_{j}^{0}$ have to do with the intrinsic stretch and twist of phase $j$ with respect to some reference state. We choose the reference state as the bottom of the well in phase $L$, so that $\lambda_{L}^{0}=1$ and $\kappa_{L}^{0}=0$. To simplify matters we choose $S_{L}=S_{H}=S, g_{L}=g_{H}=g$ and $C_{L}=C_{H}=C$. For the high strain phase we choose $\lambda_{H}^{0}=2$ and $\kappa_{H}^{0}=1$. This type of Helmholtz free energy density is illustrated in figure 2 . The two phases are separated by a line on the $\lambda-\kappa$ plane. The equation of the line is:

$$
A \lambda+B \kappa+D=0
$$

where

$$
\begin{aligned}
A & =S\left(\lambda_{H}^{0}-\lambda_{L}^{0}\right)+g\left(\kappa_{H}^{0}-\kappa_{L}^{0}\right), \\
B & =g\left(\lambda_{H}^{0}-\lambda_{L}^{0}\right)+C\left(\kappa_{H}^{0}-\kappa_{L}^{0}\right), \\
D & =\frac{S}{2}\left(\left(\lambda_{L}^{0}\right)^{2}-\left(\lambda_{H}^{0}\right)^{2}\right)+\frac{C}{2}\left(\left(\kappa_{L}^{0}\right)^{2}-\left(\kappa_{H}^{0}\right)^{2}\right)+g\left(\lambda_{L}^{0} \kappa_{L}^{0}-\lambda_{H}^{0} \kappa_{H}^{0}\right) .
\end{aligned}
$$

Here we denote $A \lambda+B \kappa+D \geq 0$ as the ' $\mathrm{H}$ ' phase and $A \lambda+B \kappa+D \leq 0$ as the 'L' phase. The force and
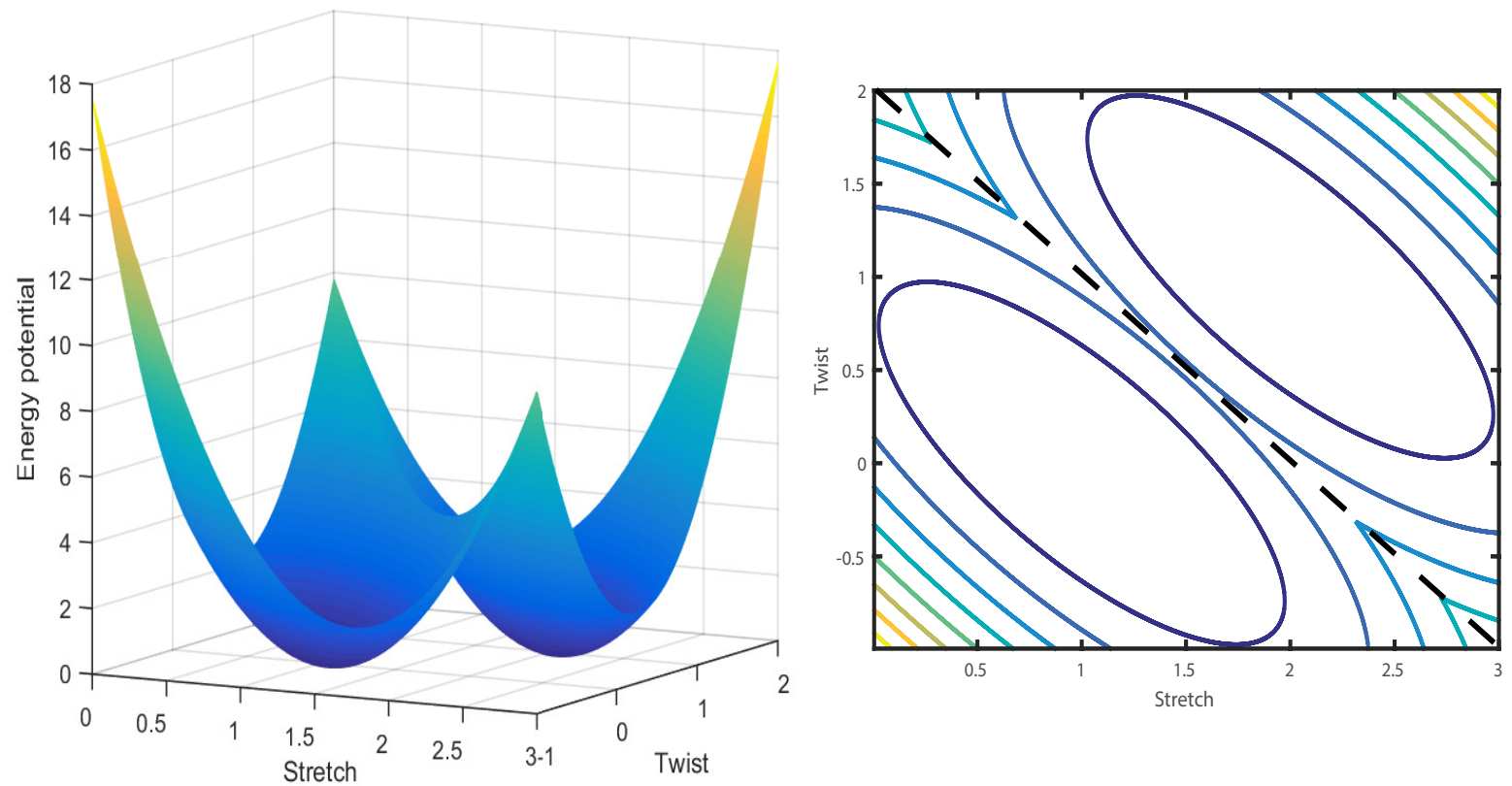

Figure 2: Energy landscape of the rod. (a) Helmholtz free energy per unit length plotted as a function of stretch $\lambda$ and twist curvature $\kappa$. It has two wells. (b) The location of the two wells shown on a contour plot in the $\lambda-\kappa$ plane. The dashed line which separates the two phases is described by the equation $A \lambda+B \kappa+D=0$. 
moment constitutive relations can be immediately computed by differentiating the $W(\lambda, \kappa)$. These are

$$
T(\lambda, \kappa)= \begin{cases}S\left(\lambda-\lambda_{L}^{0}\right)+g\left(\kappa-\kappa_{L}^{0}\right), & \text { for the 'L' phase, } \\ S\left(\lambda-\lambda_{H}^{0}\right)+g\left(\kappa-\kappa_{H}^{0}\right), & \text { for the 'H' phase, }\end{cases}
$$

and

$$
M(\lambda, \kappa)= \begin{cases}g\left(\lambda-\lambda_{L}^{0}\right)+C\left(\kappa-\kappa_{L}^{0}\right), & \text { for the 'L' phase, } \\ g\left(\lambda-\lambda_{H}^{0}\right)+C\left(\kappa-\kappa_{H}^{0}\right), & \text { for the 'H' phase. }\end{cases}
$$

\subsection{Sonic waves}

For this kind of material three kinds of discontinuities are possible - two kinds of 'sonic waves' with both sides of the discontinuity in the same phase, and a phase boundary with the two sides in different phases. Let us first consider a sonic wave type of discontinuity whose speed in the reference configuration is denoted by $c$. At such a discontinuity the jump conditions give

$$
\begin{aligned}
c\left(\lambda_{+}-\lambda_{-}\right)+v_{+}-v_{-} & =0, \\
c\left(\kappa_{+}-\kappa_{-}\right)+w_{+}-w_{-} & =0, \\
c \rho\left(v_{+}-v_{-}\right)+S\left(\lambda_{+}-\lambda_{-}\right)+g\left(\kappa_{+}-\kappa_{-}\right) & =0, \\
c \rho r_{g}^{2}\left(w_{+}-w_{-}\right)+g\left(\lambda_{+}-\lambda_{-}\right)+C\left(\kappa_{+}-\kappa_{-}\right) & =0 .
\end{aligned}
$$

We eliminate $v_{+}-v_{-}$between the first and third equations, and $w_{+}-w_{-}$between the second and fourth equations to get

$$
\begin{array}{r}
-c^{2} \rho\left(\lambda_{+}-\lambda_{-}\right)+S\left(\lambda_{+}-\lambda_{-}\right)+g\left(\kappa_{+}-\kappa_{-}\right)=0, \\
-c^{2} \rho r_{g}^{2}\left(\kappa_{+}-\kappa_{-}\right)+g\left(\lambda_{+}-\lambda_{-}\right)+C\left(\kappa_{+}-\kappa_{-}\right)=0 .
\end{array}
$$

This system of equations can have a non-trivial solution for $\lambda_{+}-\lambda_{-}$and $\kappa_{+}-\kappa_{-}$only if

$$
\frac{S-\rho c^{2}}{g}=\frac{g}{C-\rho r_{g}^{2} c^{2}}, \quad \text { or, } \quad \rho^{2} r_{g}^{2} c^{4}-\rho\left(S r_{g}^{2}+C\right) c^{2}+S C-g^{2}=0 .
$$

This is a quadratic equation for $c^{2}$ of which there are two solutions:

$$
\begin{aligned}
& c_{1}=\sqrt{\frac{\rho r_{g}^{2} S+\rho C+\sqrt{\left(\rho r_{g}^{2} S-\rho C\right)^{2}+4 \rho^{2} g^{2} r_{g}^{2}}}{2 \rho^{2} r_{g}^{2}}}, \\
& c_{2}=\sqrt{\frac{\rho r_{g}^{2} S+\rho C-\sqrt{\left(\rho r_{g}^{2} S-\rho C\right)^{2}+4 \rho^{2} g^{2} r_{g}^{2}}}{2 \rho^{2} r_{g}^{2}}} .
\end{aligned}
$$

We have chosen $c_{1}>c_{2}$. Note that $c_{1}$ would be the bar wave speed $\sqrt{S / \rho}$ and $c_{2}$ would be the torsional wave speed $\sqrt{C / \rho r_{g}^{2}}$ if the twist-stretch coupling modulus $g=0$. 


\subsection{Continuum impact problem with no phase boundary}

We will now use the jump conditions given above to solve an impact problem. Consider a semi-infinite domain, $-\infty<x \leq 0$. At $t=0, \lambda(x, 0)=\lambda_{L}, \kappa(x, 0)=\kappa_{L}, v(x, 0)=v_{L}$ and $w(x, 0)=w_{L}$ for all $x<0$. We apply the boundary conditions $v(0, t)=v_{o}$ and $w(0, t)=w_{o}$ for all $t>0$. This problem has a piece-wise constant solution with two propagating discontinuities as shown in the $\mathrm{x}$-t plane depicted in figure 3 . These discontinuities travel at speeds $c_{1}$ and $c_{2}$ if $v_{o}$ and $w_{o}$ are so small that no phase change occurs.

In the regions of the $\mathrm{x}$ - $\mathrm{t}$ plane between discontinuities the quantities $\lambda(x, t), \kappa(x, t), v(x, t)$ and $w(x, t)$ are constant. Hence, they trivially satisfy the field equations (5) and (8). At the propagating discontinuities we have to enforce the jump conditions. These are summarized below.

$$
\begin{aligned}
c_{1}\left(\lambda_{1}-\lambda_{L}\right)+v_{1}-v_{L} & =0, \\
c_{1}\left(\kappa_{1}-\kappa_{L}\right)+w_{1}-w_{L} & =0, \\
c_{1} \rho\left(v_{1}-v_{L}\right)+S\left(\lambda_{1}-\lambda_{L}\right)+g\left(\kappa_{1}-\kappa_{L}\right) & =0, \\
c_{2}\left(\lambda_{2}-\lambda_{1}\right)+v_{0}-v_{1} & =0, \\
c_{2}\left(\kappa_{2}-\kappa_{1}\right)+w_{0}-w_{1} & =0, \\
c_{2} \rho\left(v_{0}-v_{1}\right)+S\left(\lambda_{2}-\lambda_{1}\right)+g\left(\kappa_{2}-\kappa_{1}\right) & =0 .
\end{aligned}
$$

These are six equations for the six unknowns $\lambda_{1}, \kappa_{1}, v_{1}, w_{1}, \lambda_{2}, \kappa_{2}$. Note that there are three jump conditions at each of the two discontinuities. The fourth jump condition at each discontinuity does not give us any extra information because we have already used it to get the speeds $c_{1}$ and $c_{2}$. After solving this set of equations we got the analytical result for $\lambda_{1}, \kappa_{1}, v_{1}, w_{1}, \lambda_{2}, \kappa_{2}$.

In the next section we will see that this type of solution can be recovered by discretizing the continuum as a mass-spring chain with an extensional and rotational degree of freedom at each mass and solving the dynamical problem numerically. We will also see propagating phase boundaries when $v_{o}$ or $w_{o}$ are sufficiently large.

\section{Mass-spring chain}

\subsection{Description and time integration algorithm}

We have considered the dynamics of a discrete system of masses and springs to obtain the kinetics of moving phase boundaries in bars earlier [36]. The masses interacted with their first-nearest neighbors via potentials $\phi_{1}\left(x_{i}\right)$ where $x_{i}=u_{i+1}-u_{i}$ and $u_{i}$ is the displacement of the $i^{\text {th }}$ mass. The function $\phi_{1}(x)$ was a double well potential such that the equilibrium spacing between the masses at zero force could have two possible values, $a$ and $b$. The potential was constructed by using piece-wise quadratic expressions, so that the force-extension relation for each spring was trilinear (with spinodal region). We now propose that mass $i$ has a rotational degree of freedom $\theta_{i}(t)$ as well. We will call $x_{i}=u_{i+1}-u_{i}$ and $y_{i}=\theta_{i+1}-\theta_{i}$. Each mass 
interacts with its first nearest neighbor through a potential $\phi_{1}\left(x_{i}, y_{i}\right)$. Just like before, the potential $\phi$ as a function of the stretch and twist variables $x_{i}$ and $y_{i}$ has two wells representing two phases (but with no spinodal region). In each well we will assume that $\phi_{1}(x, y)$ is a quadratic form:

$$
\phi_{1}(x, y)= \begin{cases}\frac{B_{1}}{2}\left(x-a_{1}\right)^{2}+B_{2}\left(x-a_{1}\right)\left(y-b_{1}\right)+\frac{B_{3}}{2}\left(y-b_{1}\right)^{2}, & \text { in phase L, } \\ \frac{B_{1}}{2}\left(x-a_{2}\right)^{2}+B_{2}\left(x-a_{2}\right)\left(y-b_{2}\right)+\frac{B_{3}}{2}\left(y-b_{2}\right)^{2}, & \text { in phase H, }\end{cases}
$$

where the well corresponding to phase $\mathrm{L}$ is located at $\left(a_{1}, b_{1}\right)$ on the $x_{i}-y_{i}$ plane (for all $i$ ) and the well corresponding to phase $\mathrm{H}$ is located at $\left(a_{2}, b_{2}\right)$. The constant $B_{1}$ is related to the stretch modulus $S, B_{2}$ to the twist-stretch coupling modulus $g$, and $B_{3}$ to the twisting modulus $C$ as follows. Assume that the reference length for a unit in mass spring chain is $\Delta$, then $S=\Delta B_{1}, g=\Delta B_{2}, C=\Delta B_{3}$ and $\lambda_{L}^{0}=a_{1} / \Delta, \lambda_{H}^{0}=a_{2} / \Delta$, $\kappa_{L}^{0}=b_{1} / \Delta, \kappa_{H}^{0}=b_{2} / \Delta$. This results in linear relations between the force/torque and stretch/twist. The total energy of the chain consisting of $N$ masses connected by $N-1$ springs can be written as:

$$
\phi\left(u_{1}, u_{2}, \ldots, u_{N}, \theta_{1}, \theta_{2}, \ldots, \theta_{N}\right)=\sum_{i=1}^{N-1} \phi_{1}\left(x_{i}, y_{i}\right) .
$$

The dynamics of the chain is governed by the following system of ordinary differential equations:

$$
m_{i} \ddot{u}_{i}=\frac{\partial \phi}{\partial u_{i}}, \quad I_{i} \ddot{\theta}_{i}=\frac{\partial \phi}{\partial \theta_{i}}, \quad i=1, \ldots, N .
$$

Here $m_{i}$ is the $i^{\text {th }}$ mass and $I_{i}$ is the moment of inertia of the $i^{\text {th }}$ mass about the straight line connecting all the masses. We assume for simplicity that $m_{i}=m$ and $I_{i}=I$ for all $i$. The evolution of the variables $u_{i}(t)$ and $\theta_{i}(t)$ can be calculated by solving the above system of differential equations subject to some initial conditions $u_{i}(0)=u_{i}^{0}, \dot{u}_{i}(0)=v_{i}^{0}, \theta_{i}(0)=\theta_{i}^{0}, \dot{\theta}_{i}(0)=w_{i}^{0}, i=1, \ldots, N$ and some boundary conditions on $u_{0}(t), \theta_{0}(t)$ and $u_{N}(t), \theta_{N}(t)$. Here, $u_{i}^{0}$ is the $i^{t h}$ component of a given vector $u^{0}$ with $N$ entries, and similarly for $v_{i}^{0}, \theta_{i}^{0}, w_{i}^{0}$.

The equations of motion of the masses for $i=2, \ldots, N-1$ can be written as

$$
\begin{aligned}
& m \ddot{u}_{i}=T_{i+\frac{1}{2}}-T_{i-\frac{1}{2}}, \\
& I \ddot{\theta}_{i}=M_{i+\frac{1}{2}}-M_{i-\frac{1}{2}},
\end{aligned}
$$

where $i+\frac{1}{2}$ and $i-\frac{1}{2}$ denote the springs attached to mass $i$. Here $T_{i+\frac{1}{2}}$ is the force in the spring connecting masses $i+1$ and $i$, etc. Without loss of generality we can take $m=1$ and $I=1$, then

$$
\begin{gathered}
\ddot{u}_{i}=T\left(u_{i+1}-u_{i}, \theta_{i+1}-\theta_{i}\right)-T\left(u_{i}-u_{i-1}, \theta_{i}-\theta_{i-1}\right), \\
\ddot{\theta}_{i}=M\left(u_{i+1}-u_{i}, \theta_{i+1}-\theta_{i}\right)-M\left(u_{i}-u_{i-1}, \theta_{i}-\theta_{i-1}\right) .
\end{gathered}
$$

We use a leapfrog algorithm to integrate the system. We start from an initial condition in which we know the position and velocity (both linear and angular) of each mass, then we carry out our calculation 
by updating the state of each mass as follows.

$$
\begin{array}{r}
T_{j+\frac{1}{2}}^{i}=B_{1}\left(x_{j+\frac{1}{2}}^{i}-a_{k}\right)+B_{2}\left(y_{j+\frac{1}{2}}^{i}-b_{k}\right), \\
M_{j+\frac{1}{2}}^{i}=B_{2}\left(x_{j+\frac{1}{2}}^{i}-a_{k}\right)+B_{3}\left(y_{j+\frac{1}{2}}^{i}-b_{k}\right), \\
u_{j}^{i+1}=u_{j}^{i}+\Delta t v_{j}^{i}+\frac{1}{2} \Delta t^{2}\left(T_{j+\frac{1}{2}}^{i}-T_{j-\frac{1}{2}}^{i}\right), \\
\theta_{j}^{i+1}=\theta_{j}^{i}+\Delta t w_{j}^{i}+\frac{1}{2} \Delta t^{2}\left(M_{j+\frac{1}{2}}^{i}-M_{j-\frac{1}{2}}^{i}\right), \\
v_{j}^{i+1}=v_{j}^{i}+\frac{1}{2} \Delta t\left(T_{j+\frac{1}{2}}^{i}-T_{j-\frac{1}{2}}^{i}+T_{j+\frac{1}{2}}^{i+1}-T_{j-\frac{1}{2}}^{i+1}\right), \\
w_{j}^{i+1}=w_{j}^{i}+\frac{1}{2} \Delta t\left(M_{j+\frac{1}{2}}^{i}-M_{j-\frac{1}{2}}^{i}+M_{j+\frac{1}{2}}^{i+1}-M_{j-\frac{1}{2}}^{i+1}\right) .
\end{array}
$$

Here the lower index denotes the mass number, the upper index denotes the time step, and $k=1$ or 2 , depending on whether the spring is in the ' $\mathrm{L}$ ' phase of ' $\mathrm{H}$ ' phase. We choose a time step $\Delta t$ that is much smaller than $a_{1} / c_{1}$ to ensure that our numerical calculation is stable. Recall that $a_{1}$ is the distance between masses (or the lattice parameter in the ' $L$ ' phase) when no forces are applied on the chain.

\subsection{Impact problem}

Now, we would like to simulate an impact problem using our mass-spring chain. For impact problems, ideally the domain is semi-infinite. In our calculations, we approximate the problem as a mass-spring chain with $N$ masses and $N-1$ springs connecting them. We take $N=100$ and fix the left end of the chain $(j=1)$ and apply constant linear and angular velocity at the right end $(j=N)$. Hence, $u_{1}^{i}=0, \theta_{1}^{i}=0$ and $u_{N}^{i}=v_{0} i \Delta t+u_{N}^{0}, \theta_{N}^{i}=w_{0} i \Delta t+\theta_{N}^{0}$, where $i$ is the time-step number. Initially, all springs are in the ' $\mathrm{L}$ ' phase. We integrate equations 26 for the system up to the point that the faster sonic wave which originated at $j=N$ hits the left end $(j=1)$. We find that below some critical set of values of applied velocities $v_{0}, w_{0}$, there are only two sonic waves passing through the chain from right to left. The sonic waves travel at constant speeds $c_{1}$ and $c_{2}$ as given in equation 19 and shown in figure 3. The oscillatory strains and velocities behind these waves decay to constants as expected from the analytical solution of the impact problem. If we apply higher extensional and angular velocities, a phase transition nucleates at $j=N$ and the phase boundary propagates inwards towards $j=1$ as shown in figure 4 with velocity $\dot{s}$. We initially use the material parameter set $B_{1}=10, B_{2}=7.6$ and $B_{3}=10$ for our numerical calculation. However, we have also used other material parameter combinations like $B_{1}=10, B_{2}=3, B_{3}=10$ and $B_{1}=100, B_{2}=8, B_{3}=10$ and have confirmed that the results are similar. We have observed in our numerical simulations that phase boundary speed $\dot{s}$ is always smaller than $c_{1}$. We find, interestingly, that for most material parameter sets $B_{1}, B_{2}, B_{3}$, the phase boundary velocity is larger than the slower sonic wave speed $c_{2}$, no matter what boundary condition we apply. For some material parameter sets we get phase boundary speeds smaller than $c_{2}$, but surprisingly the dissipation rates for these cases, as calculated using isothermal expressions for the driving force, are negative. We will consider this case later. First, we consider 

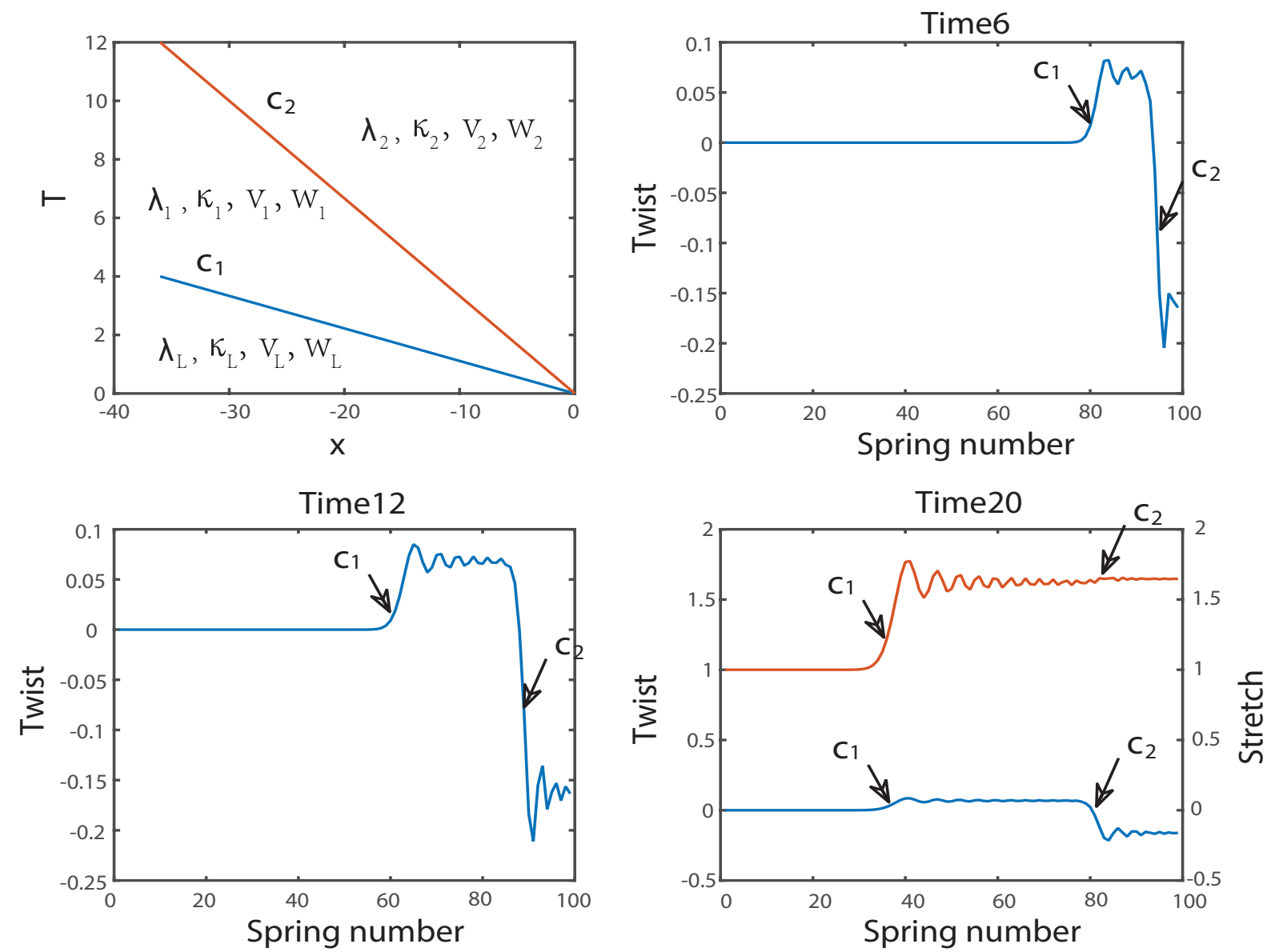

Figure 3: An example of an impact problem with only two sonic waves. On the top left is the $x-t$ plane for the problem. The remaining three panels show the twist as a function of spring number at three different times. Two discontinuities with a jump in twist are clearly seen. They move at speeds $c_{1}$ and $c_{2}$. The bottom right panel also shows the stretch as a function of spring number, again revealing two waves.

the situation in which $c_{1}>\dot{s}>c_{2}$. As we can see from the $x-t$ plane in figure 4 and the dynamics of the chain, there are three moving discontinuities and piecewise constant strain and velocity profiles between these discontinuities. Note, however, that the strains and velocities behind the phase boundary do not decay, but oscillate around constant values. It is these constant strains and velocities behind the phase boundary that will enter the jump conditions. For the continuum impact problem with a single phase boundary we 


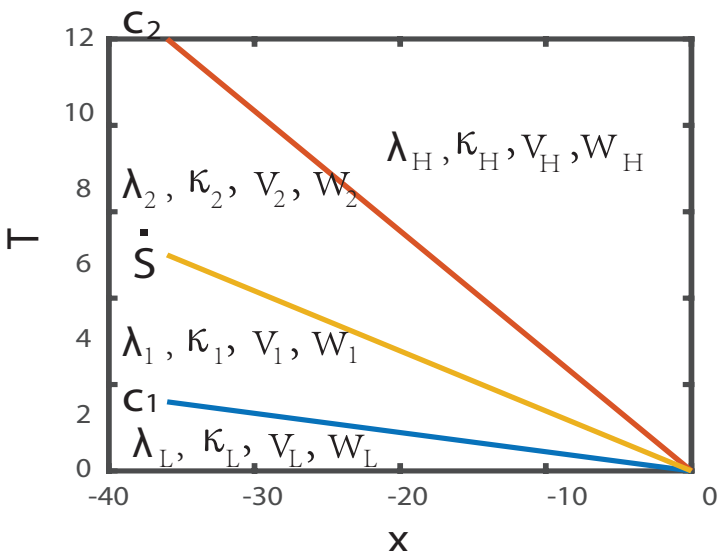

Time12

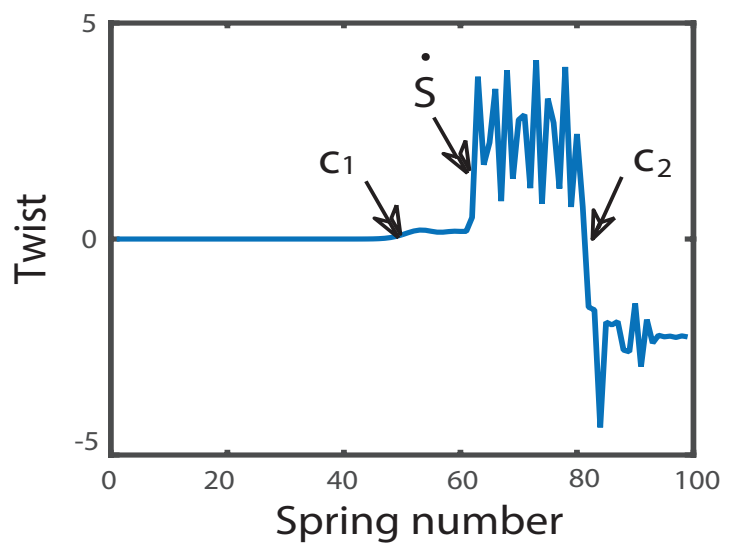

Time6

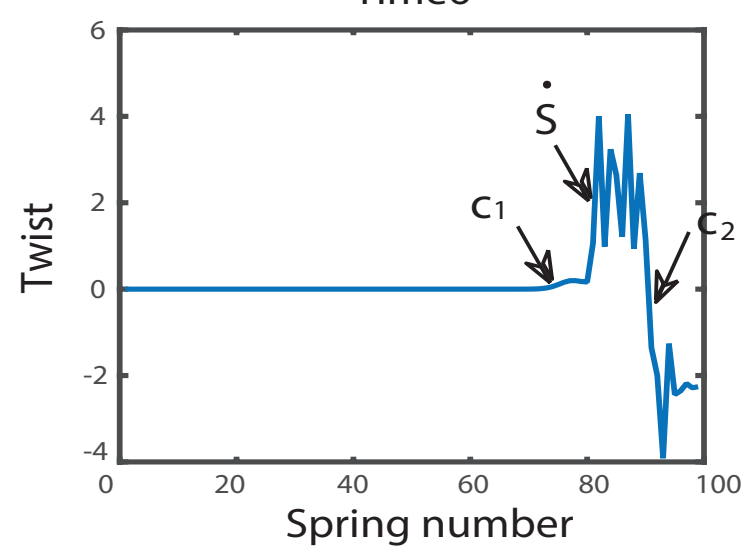

Time20

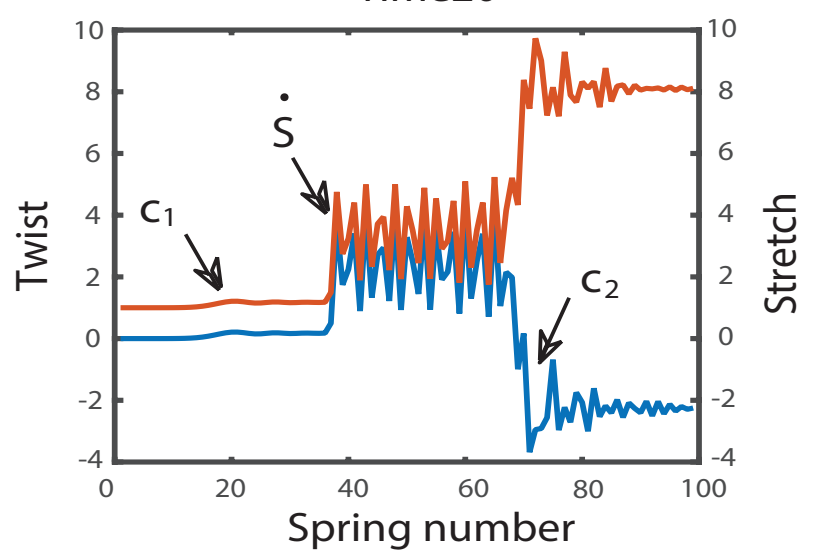

Figure 4: An example of an impact problem with a single phase boundary. The top left panel shows the $x-t$ plane. Three waves are present, two sonic waves at speeds $c_{1}$ and $c_{2}$, and a phase boundary at speed $\dot{s}$ with $c_{1}>\dot{s}>c_{2}$. The rest of the panels show snapshots of the twist in the chain as function of spring number at three different times. The bottom right panel also shows a snapshot of the stretch, again showing three discontinuities.

can now write down the jump conditions:

$$
\begin{aligned}
c_{1}\left(\lambda_{1}-\lambda_{L}\right)+v_{1}-v_{L} & =0, \\
c_{1}\left(\kappa_{1}-\kappa_{L}\right)+w_{1}-w_{L} & =0, \\
c_{1}\left(v_{1}-v_{L}\right)+S\left(\lambda_{1}-\lambda_{L}\right)+g\left(\kappa_{1}-\kappa_{L}\right) & =0, \\
c_{2}\left(\lambda_{H}-\lambda_{2}\right)+v_{H}-v_{2} & =0, \\
c_{2}\left(\kappa_{H}-\kappa_{2}\right)+w_{H}-w_{2} & =0, \\
S\left(\lambda_{H}-\lambda_{2}\right)+g\left(\kappa_{H}-\kappa_{2}\right) & =0, \\
\dot{s}\left(\lambda_{2}-\lambda_{1}\right)+v_{2}-v_{1} & =0, \\
\dot{s}\left(\kappa_{2}-\kappa_{1}\right)+w_{2}-w_{1} & =0, \\
S\left(\lambda_{2}-\lambda_{1}\right)+g\left(\kappa_{2}-\kappa_{1}\right) & =0, \\
\left.\dot{s}\left(v_{2}-v_{1}\right)+w_{1}\right)+g\left(\lambda_{2}-\lambda_{1}-\lambda_{H}^{0}+\lambda_{L}^{\mathrm{b}}\right)+C\left(\kappa_{2}-\kappa_{1}-\kappa_{H}^{0}+\kappa_{L}^{0}\right) & =0 .
\end{aligned}
$$


We have ten equations for the eleven unknowns $\lambda_{1}, \kappa_{1}, v_{1}, w_{1}, \lambda_{2}, \kappa_{2}, v_{2}, w_{2}, \lambda_{H}, \kappa_{H}, \dot{s}$. These jump conditions are not sufficient to describe the motion of the phase boundary or the dynamics of the system. We need a kinetic relation which relates phase boundary velocity and driving force to close the analysis. We will extract this kinetic relation from the simulations of our mass-spring system.

\subsection{Kinetic relation}

We have carried out a series of computations for impact problems with different material sets $B_{1}, B_{2}, B_{3}$. For each set we have applied several extensional and rotational velocities (or boundary conditions) and in each case we have obtained quantities like $\lambda_{1}, \kappa_{1}, v_{1}, w_{1}, \lambda_{2}, \kappa_{2}, v_{2}, w_{2}, \lambda_{H}, \kappa_{H}, \dot{s}$ by post-processing the numerical result. We then compute the driving force as defined in equation 9 and normalized phase boundary velocity $\dot{s} / c_{1}$. For each material set we plot the driving force versus phase boundary velocity to find a relation between them. If we normalize the driving force suitably then all the curves of driving force versus phase boundary velocity can be described by the equation 28 which is the kinetic relation we wanted. The normalizing factor for the driving force depends on the material parameter set.

$$
\frac{\left(\frac{c_{2}}{c_{1}}\right)^{2}-\left(1-\left(\frac{c_{2}}{c_{1}}\right)^{2}\right) f_{\text {threshold }}-\left(\frac{\dot{s}}{c_{1}}\right)^{2}}{1-\left(\frac{\dot{s}}{c_{1}}\right)^{2}}=f_{\text {normalized }}=\frac{[|W|]-\langle T\rangle[|\lambda|]-\langle M\rangle[|\kappa|]}{\frac{2}{l_{0}} \sqrt{S C+g^{2}}} .
$$

In this kinetic relation, $\frac{2}{l_{0}} \sqrt{S C+g^{2}}$ is the normalizing factor for the driving force, $f_{\text {threshold }}$ is the threshold value of driving force when the phase transition just begins to happen and $l_{0}=\frac{\lambda_{H}^{0}-\lambda_{L}^{0}}{\kappa_{H}^{0}-\kappa_{L}^{0}}$. This kinetic relation has the familiar form

$$
f_{\text {driving }}=q_{1}+\frac{q_{2}}{1-\frac{\dot{s}^{2}}{c_{1}^{2}}},
$$

where $q_{1}$ and $q_{2}$ are constants that depend on the chosen material parameters. It can be rearranged in the following manner

$$
\frac{1}{1-\left(\frac{\dot{s}}{c_{1}}\right)^{2}}=\frac{1-\frac{l_{0}}{2 \sqrt{S C+g^{2}}}([|W|]-\langle T\rangle[|\lambda|]-\langle M\rangle[|\kappa|])}{\left(1+f_{\text {threshold }}\right)\left(1-\left(\frac{c_{2}}{c_{1}}\right)^{2}\right)} .
$$

The RHS of this equation is plotted on the x-axis of figure 5 and $\dot{s} / c_{1}$ is plotted on the y-axis. We have solved the continuum impact problem analytically by using the kinetic relation equation 30 . The result is shown in table 1 and figure 8 for two material parameter sets. We see from the table that the analytical result is consistent with the numerical result. This suggests that the kinetic relation we have extracted can be used to solve other types of problems.

\subsection{Analytical and numerical solutions of Riemann problem}

Riemann problems in bars with a single phase boundary have been treated earlier [1,22]. It is well understood that for the type of constitutive relations we use in this work the solutions of these problems are piecewise constant in the $\mathrm{x}$-t plane. The domain for a Riemann problem is $-\infty<x<\infty$. However, since 


\begin{tabular}{c|c|c|c|c|c|c|c|c|c}
\hline$v_{0}$ & 4.5 & 8 & 6 & 10 & 12 & 16 & 20 & 30 & 50 \\
\hline$w_{0}$ & 1 & 1 & 0 & 0 & 0 & 0 & 0 & 0 & 0 \\
\hline Analytical $\dot{s}$ & -1.7 & -2.205 & -1.7764 & -2.505 & -2.7368 & -3.0512 & -3.2528 & -3.5361 & -4.1642 \\
\hline Numerical $\dot{s}$ & -1.75 & -2.35 & -1.95 & -2.55 & -2.8 & -3.1 & -3.3 & -3.55 & -4.15 \\
\hline Analytical $\lambda_{1}$ & 0.1679 & 0.2546 & 0.1992 & 0.2639 & 0.2946 & 0.3628 & 0.4394 & 0.6643 & 0.8636 \\
\hline Numerical $\lambda_{1}$ & 0.1537 & 0.1783 & 0.142 & 0.161 & 0.1683 & 0.1785 & 0.1862 & 0.1987 & 0.1974 \\
\hline Analytical $\lambda_{2}$ & 1.2473 & 1.741 & 1.4176 & 1.818 & 2.0354 & 2.4859 & 2.9469 & 4.1181 & 6.4593 \\
\hline Numerical $\lambda_{2}$ & 1.1545 & 1.76 & 1.41 & 1.86 & 2.024 & 2.479 & 2.972 & 4.048 & 6.461 \\
\hline Analytical $\lambda_{H}$ & 2.7247 & 4.754 & 3.3541 & 5.0454 & 5.9084 & 7.6498 & 9.4018 & 13.8005 & 22.5999 \\
\hline Numerical $\lambda_{H}$ & 2.685 & 4.758 & 3.279 & 5.058 & 5.94 & 7.652 & 9.432 & 13.81 & 22.65 \\
\hline Analytical $\kappa_{1}$ & 0.1679 & 0.2546 & 0.1992 & 0.2639 & 0.2945 & 0.3628 & 0.4394 & 0.6643 & 0.8636 \\
\hline Numerical $\kappa_{1}$ & 0.1537 & 0.1783 & 0.142 & 0.161 & 0.1683 & 0.1785 & 0.1862 & 0.1987 & 0.1974 \\
\hline Analytical $\kappa_{2}$ & 1.2473 & 1.741 & 1.4176 & 1.818 & 2.0354 & 2.4859 & 2.9469 & 4.1181 & 6.4593 \\
\hline Numerical $\kappa_{2}$ & 1.1545 & 1.76 & 1.072 & 1.857 & 2.02 & 2.479 & 3.018 & 4.03 & 6.461 \\
\hline Analytical $\kappa_{H}$ & -0.195 & -1.295 & -0.5189 & -1.4095 & -1.8376 & -2.6781 & -3.508 & -5.5643 & -9.6789 \\
\hline Numerical $\kappa_{H}$ & -0.2256 & -1.312 & -0.6016 & -1.416 & -1.82 & -2.695 & -3.542 & -5.595 & -9.691 \\
\hline
\end{tabular}

Table 1: Comparison of numerical results with analytical solution of impact problems for $B_{1}=10, B_{2}=7.6, B_{3}=10$. The analytical results use the kinetic relation 30. The agreement between quantities like phase boundary velocity obtained from numerical integration and continuum analytic solution is quite good, especially for large $v_{0}$. 


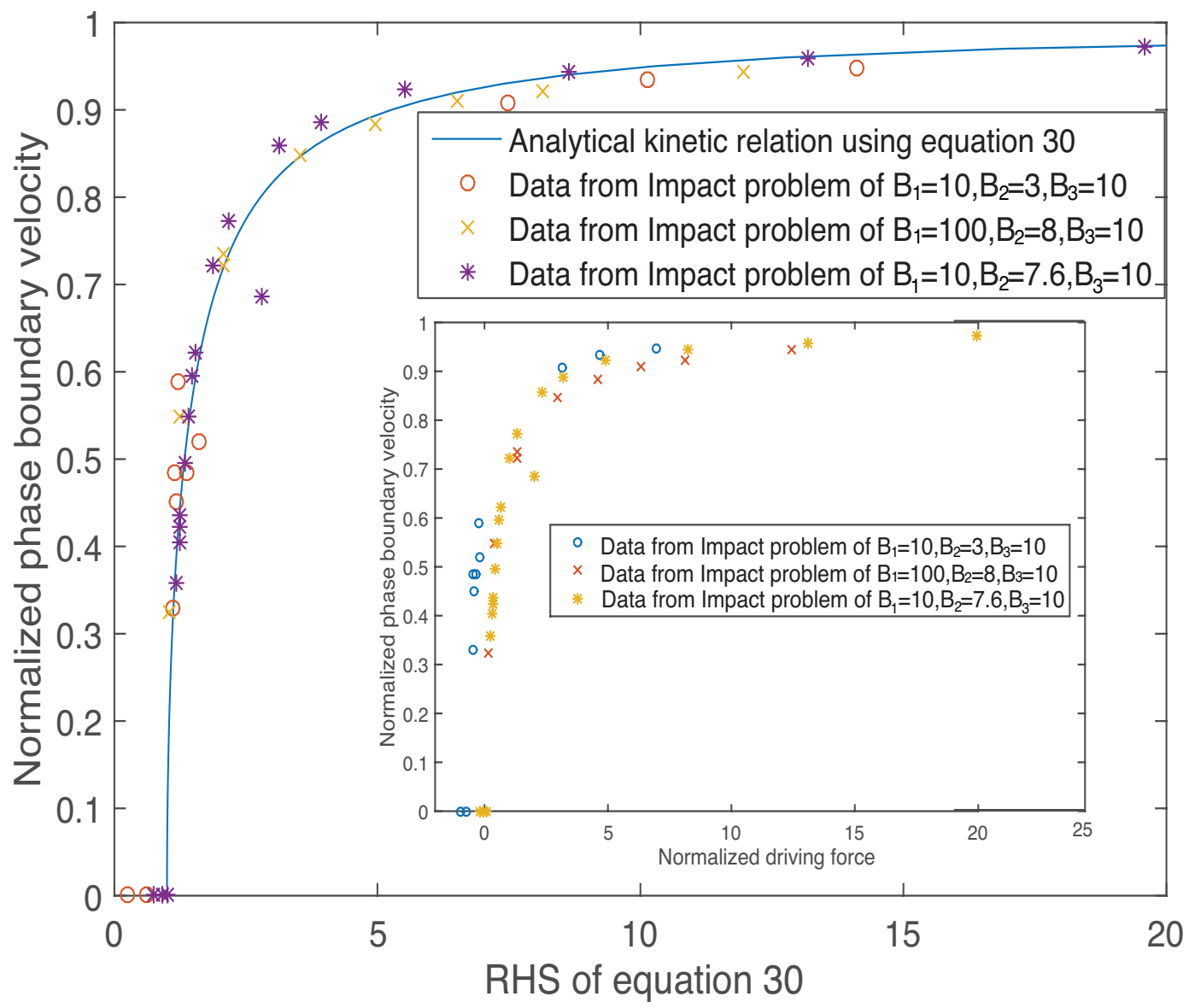

Figure 5: Kinetic relation using equation 30. The data for this plot was obtained from many simulations with different material sets for the 'springs'. Phase boundary velocity $\dot{s}$ is normalized against $c_{1}$ and plotted on the $y$-axis. Driving force $f_{d r i v i n g}$ is normalized according to equation 30 and plotted on the $x$-axis. Note that for driving forces below a threshold the phase boundary velocity is zero. The inset of the figure is the original data of normalized phase boundary velocity vs. the normalized driving force (as in equation 28). Note that some of the blue circles result in $\dot{s} f_{\text {driving }}<0$.

we cannot simulate an infinite mass-spring chain we have used $N+1$ masses and $N$ springs with an initial phase boundary in the middle of the chain at mass number $\frac{N}{2}$ where $N=1000$. The equations of motion for the masses is equation 24 and the two boundaries are left free (zero force and moment). At $t=0$ we prescribe initial conditions such that all springs on the left half are in the ' $\mathrm{L}$ ' phase, while those on the right are in the 'H' phase. Since our mass-spring system has a finite number of masses and has two boundaries there could be four additional sonic waves which originate from the two ends. We integrate the dynamics of the mass spring system up to the point when the sonic waves originating at the center of the chain and those coming from the ends meet each other. Again, we start our calculation with the material set $B_{1}=10$, $B_{2}=7.6, B_{3}=10$, but the features we observe are similar even if we use other parameter sets. When we let this system evolve we notice three different scenarios. 
In the first scenario the phase boundary in the middle propagates along the chain. An example of the evolution of such a system is shown in figure 7 . We see that there is a phase boundary moving from the middle of the chain to the left in addition to the sonic waves described above. If we focus on the middle part of the chain between the four sonic waves moving outward, then the x-t plane looks exactly like what is expected from the continuum theory as shown in figure 6 .
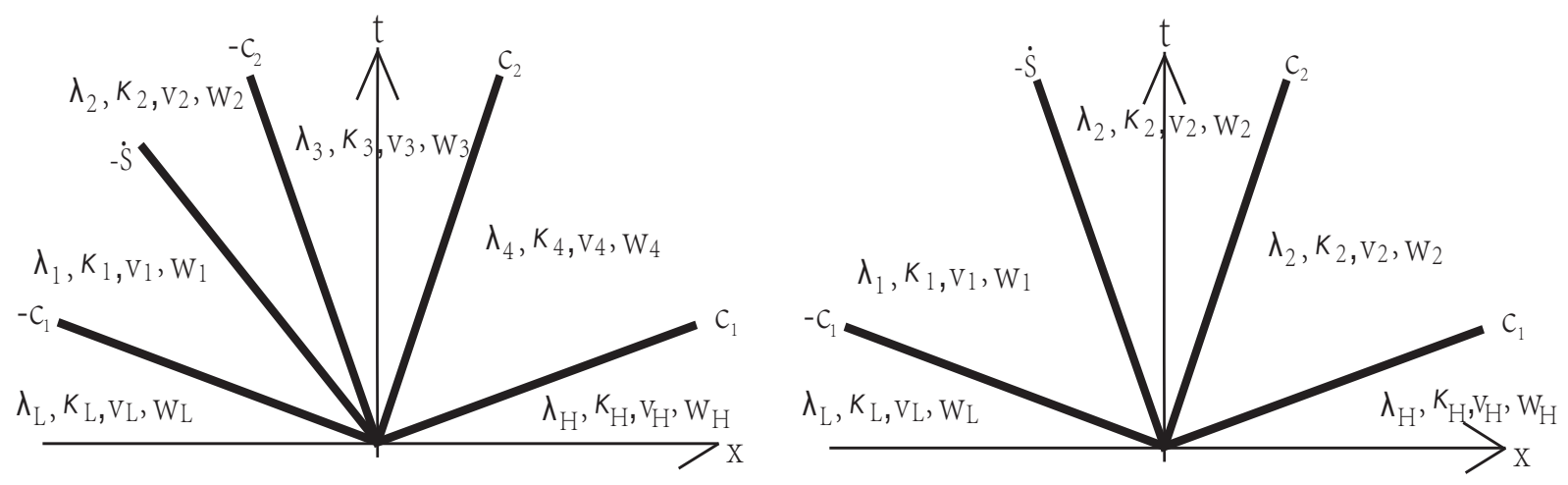

Figure 6: (a) $x-t$ plane of a Riemann problem with a single phase boundary with $c_{1}>\dot{s}>c_{2}$. (b) $x-t$ plane of a Riemann problem in which $\dot{s}=-c_{2}$.

From our simulations we find that the phase boundary speed is smaller than the larger sonic wave speed $c_{1}$, as expected. For most material parameter sets the phase boundary speed is larger than the slower sonic wave $c_{2}$ no matter what initial condition we set. However, for some material parameter sets (for example, $\left.B_{1}=10, B_{2}=3, B_{3}=10\right)$, the phase boundary speed can be smaller than $c_{2}$. In these cases the dissipation rate $f_{\text {driving }} \dot{s}$ is negative, which is physically not possible. Cases with $\dot{s}<c_{2}$ were observed in the impact problem with the same set of material parameters also. We will explain this later.

By post-processing the data from our numerical calculations we can obtain $\lambda_{1}, \kappa_{1}, v_{1}, w_{1}, \lambda_{2}, \kappa_{2}, v_{2}, w_{2}$, $\lambda_{3}, \kappa_{3}, v_{3}, w_{3}, \lambda_{4}, \kappa_{4}, v_{4}, w_{4}, \lambda_{4}, \kappa_{4}, v_{4}, w_{4}, \dot{s}$ (see figure 6 ). We compare these numerical results with those of the solution of the continuum Riemann problem that can be solved analytically using the kinetic relation 30. We assume that the initial conditions are at $t=0, \lambda=\lambda_{L}, v=v_{L}, \kappa=\kappa_{L}, w=w_{L}$ for $x<0$ and $\lambda=\lambda_{H}, v=v_{H}, \kappa=\kappa_{H}, w=w_{H}$ for $x>0$. For $t>0$ this Riemann problem has piece-wise constant solution depicted in the $\mathrm{x}$-t plane figure 6 . There are a total of seventeen unknowns $\lambda_{1}, \kappa_{1}, v_{1}, w_{1}, \lambda_{2}, \kappa_{2}, v_{2}, w_{2}$, $\lambda_{3}, \kappa_{3}, v_{3}, w_{3}, \lambda_{4}, \kappa_{4}, v_{4}, w_{4}, \lambda_{4}, \kappa_{4}, v_{4}, w_{4}, \dot{s}$. We enforce the jump conditions at each discontinuity and get sixteen equations as follows. The kinetic relation equation (30) provides the seventeenth equation. 

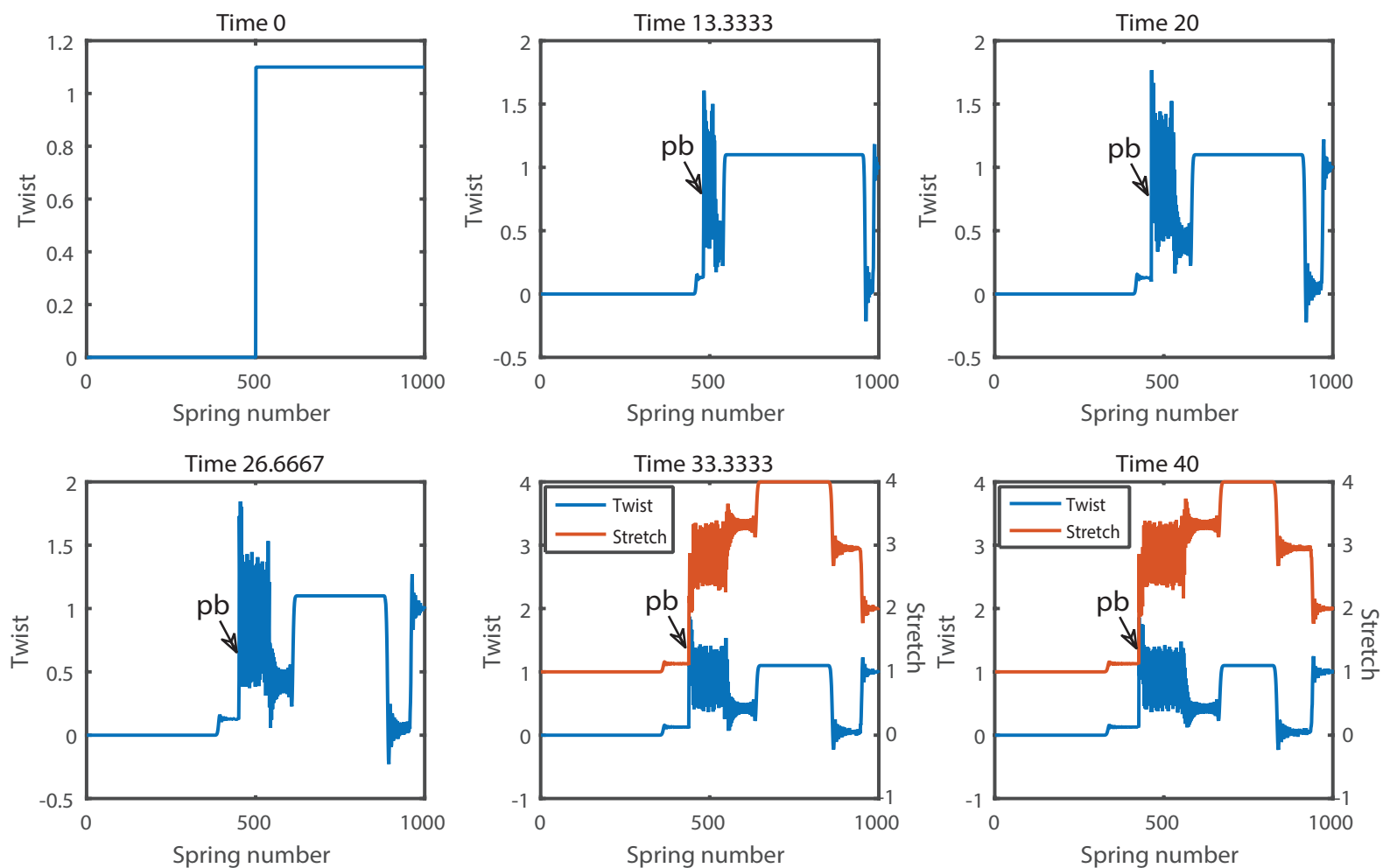

Figure 7: An example of an Riemann problem with a single phase boundary. Five waves are present, two sonic waves at speeds $c_{1}$ and $c_{2}$ propagating in opposite directions from the middle, and a phase boundary at speed $\dot{s}$ with $c_{1}>\dot{s}>c_{2}$. There are also two sonic waves moving towards the left from the right boundary. In general there should also be two sonic waves moving rightwards from the left end, but we do not see them above because of the choice of initial conditions. The panels show snapshots of the twist in the chain as function of spring number at six different times. Two bottom panels also show snapshots of the stretch.

$$
\begin{aligned}
& \text { Across }-c_{1}\left\{\begin{array}{l}
-c_{1}\left(\lambda_{1}-\lambda_{L}\right)+v_{1}-v_{L}=0, \\
-c_{1}\left(\kappa_{1}-\kappa_{L}\right)+w_{1}-w_{L}=0, \\
-c_{1}\left(v_{1}-v_{L}\right)+S\left(\lambda_{1}-\lambda_{L}\right)+g\left(\kappa_{1}-\kappa_{L}\right)=0,
\end{array}\right. \\
& \text { Across } \dot{s}\left\{\begin{array}{l}
\dot{s}\left(\lambda_{2}-\lambda_{1}\right)+v_{2}-v_{1}=0, \\
\dot{s}\left(\kappa_{2}-\kappa_{1}\right)+w_{2}-w_{1}=0, \\
\dot{s}\left(v_{2}-v_{1}\right)+S\left(\lambda_{2}-\lambda_{1}-\lambda_{H}^{0}+\lambda_{L}^{0}\right)+g\left(\kappa_{2}-\kappa_{1}-\kappa_{H}^{0}+\kappa_{L}^{0}\right)=0, \\
\dot{s}\left(w_{2}-w_{1}\right)+g\left(\lambda_{2}-\lambda_{1}-\lambda_{H}^{0}+\lambda_{L}^{0}\right)+C\left(\kappa_{2}-\kappa_{1}-\kappa_{H}^{0}+\kappa_{L}^{0}\right)=0,
\end{array}\right. \\
& \text { Across }-c_{2}\left\{\begin{array}{l}
-c_{2}\left(\lambda_{3}-\lambda_{2}\right)+v_{3}-v_{2}=0, \\
-c_{2}\left(\kappa_{3}-\kappa_{2}\right)+w_{3}-w_{2}=0, \\
-c_{2}\left(v_{3}-v_{2}\right)+S\left(\lambda_{3}-\lambda_{2}\right)+g\left(\kappa_{3}-\kappa_{2}\right)=0,
\end{array}\right.
\end{aligned}
$$



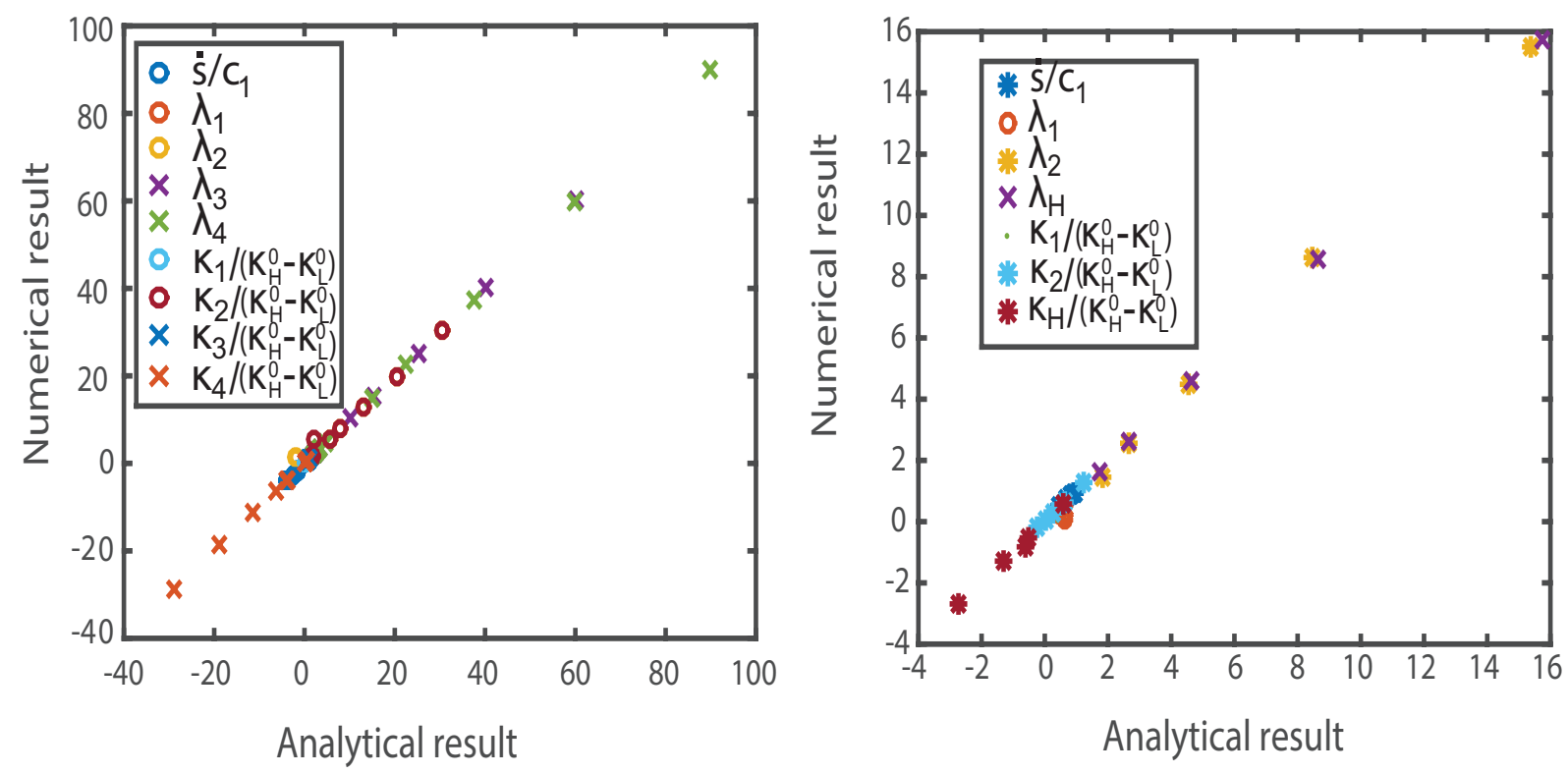

Figure 8: (a) Scatter plot showing comparison of numerical results with analytical solution of Riemann problems for $B_{1}=$ $10, B_{2}=7.6, B_{3}=10$. The analytical results use the kinetic relation obtained from impact problems on the chain to solve the set of equations 3.4. The agreement between the phase boundary velocities and other quantities obtained from the numerical integration of the equations of motion of the chain and those from the analogous continuum analytic solution is quite good. (b) Scatter plot showing comparison of numerical results with analytical solution of impact problems for $B_{1}=100, B_{2}=8, B_{3}=10$. The analytical results use the kinetic relation 30.

$$
\begin{aligned}
& \text { Across } c_{2}\left\{\begin{array}{l}
c_{2}\left(\lambda_{4}-\lambda_{3}\right)+v_{4}-v_{3}=0, \\
c_{2}\left(\kappa_{4}-\kappa_{3}\right)+w_{4}-w_{3}=0, \\
c_{2}\left(v_{4}-v_{3}\right)+S\left(\lambda_{4}-\lambda_{3}\right)+g\left(\kappa_{4}-\kappa_{3}\right)=0,
\end{array}\right. \\
& \operatorname{Across} c_{1}\left\{\begin{array}{l}
c_{1}\left(\lambda_{H}-\lambda_{4}\right)+v_{H}-v_{4}=0, \\
c_{1}\left(\kappa_{H}-\kappa_{4}\right)+w_{H}-w_{4}=0, \\
c_{1}\left(v_{H}-v_{4}\right)+S\left(\lambda_{H}-\lambda_{4}\right)+g\left(\kappa_{H}-\kappa_{4}\right)=0 .
\end{array}\right.
\end{aligned}
$$

These equations can be solved analytically using Maple or similar programs. We compare the results from this exercise with those of the numerical solution in the scatter plot figure 11 for several different initial conditions. We plot non-dimensional quantities like $\lambda_{1}, v_{1} / c_{1}$, etc., obtained from the numerical calculations on the $\mathrm{y}$-axis and those from the analytical solutions of equations 3.4 on the $\mathrm{x}$-axis. We see that points in this scatter plot make a line with slope 1 which indicates good agreement between the mass-spring chain and the analytical solution. This suggests that the kinetic relation equation (30) is indeed a useful constitutive law. 
The first scenario described above validates our kinetic relation. But, there are two more scenarios in which a kinetic relation is not needed for the solution of the Riemann problem with a single phase boundary. In the second scenario, the phase boundary in the middle remains static (a contact discontinuity), but four sonic waves with speeds $\pm c_{1}$ and $\pm c_{2}$ propagate towards the left and right. An example of the evolution of the system is given in figure 9 . In this case the continuum Riemann problem can be solved analytically
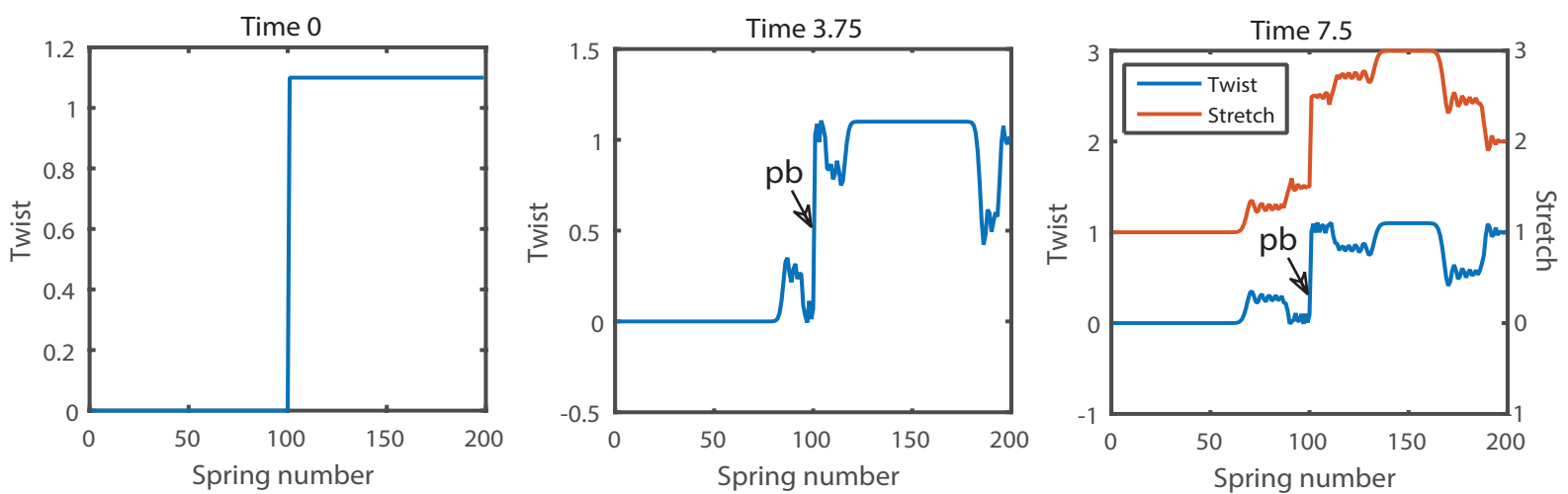

Figure 9: An example of an Riemann problem with a static phase boundary. Four waves are present, two sonic waves at speeds $c_{1}$ and $c_{2}$ propagating in opposite directions from the middle while the phase boundary at the middle remains static. Two sonic waves also move inwards from the right boundary. The panels show snapshots of the twist in the chain as function of spring number at three different times. The right panel also shows a snapshot of the stretch.

using the jump conditions alone. There are sixteen unknowns and sixteen equations to solve for them. Each sonic wave contributes three equations and the contact discontinuity contributes four. The equations are similar to equation 3.4 except that we don't need a kinetic relation since $\dot{s}=0$. Once again, the scatter plot in figure 11 compares quantities from the numerical simulation of the mass-spring chain and the analytical solution. A slope of 1 indicates excellent agreement.

In the third scenario the phase boundary propagates at the speed $c_{2}$ and becomes indistinguishable from one of the sonic waves. Thus, the impact problem with a phase boundary in this pathological case has only two discontinuities instead of three, and the Riemann problem has four discontinuities instead of five. An example of the evolution of such a system is shown in figure 10. Note that we have four waves on the $x-t$ plane with speeds $-c_{1},-\dot{s}=-c_{2}, c_{2}$ and $c_{1}$. There are twelve unknowns and twelve equations (three jump conditions at each discontinuity) to solve for them. We have found, again, that analytical and numerical solutions for this case agree quite well.

So far in our analysis we have described cases in which phase boundary velocities $\dot{s}>c_{2}$. However, when we choose a material parameter set $B_{1}=10, B_{2}=3, B_{3}=10$, we find situations in which phase boundary speed is smaller than $c_{2}$. An example of such dynamics is given in figure 13. Once again, after post-processing we have verified that the piecewise constant stretches, velocities, angular velocities and twisting curvatures satisfy all the jump conditions. Indeed, the driving force $f_{\text {driving }}$ computed using the parameters extracted 

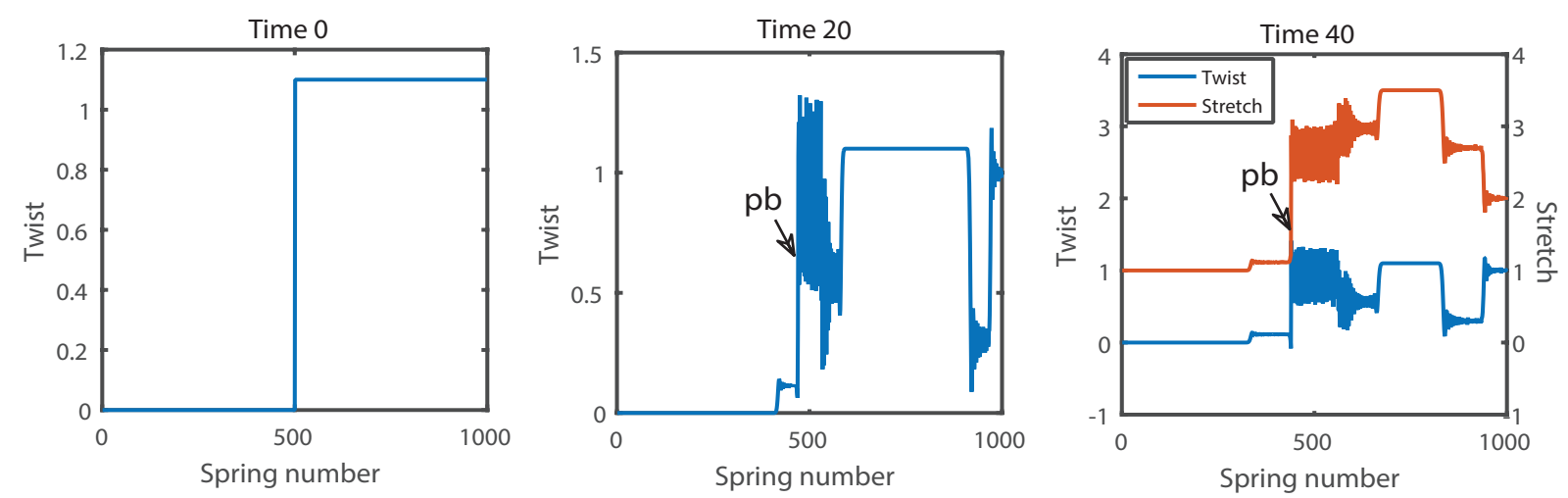

Figure 10: An example of a Riemann problem in which phase boundary merges with a $c_{2}$ sonic wave. Four waves are present, three sonic waves at speeds $c_{1}$ and $c_{2}$ propagating in opposite directions from the middle, and a phase boundary traveling at speed $c_{2}$. The panels show snapshots of the twist in the chain as function of spring number at three different times. The right panel also shows a snapshot of the stretch.

(a)

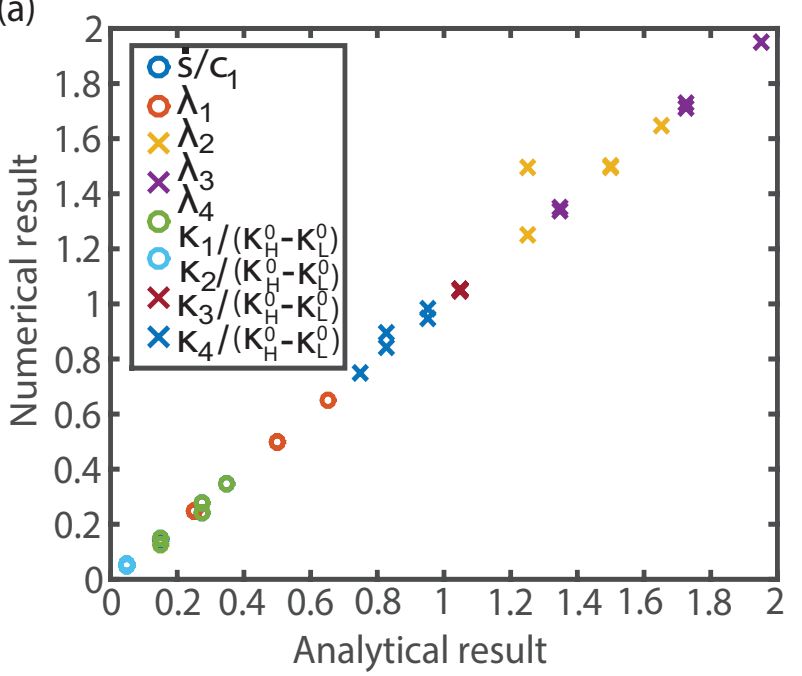

(b)

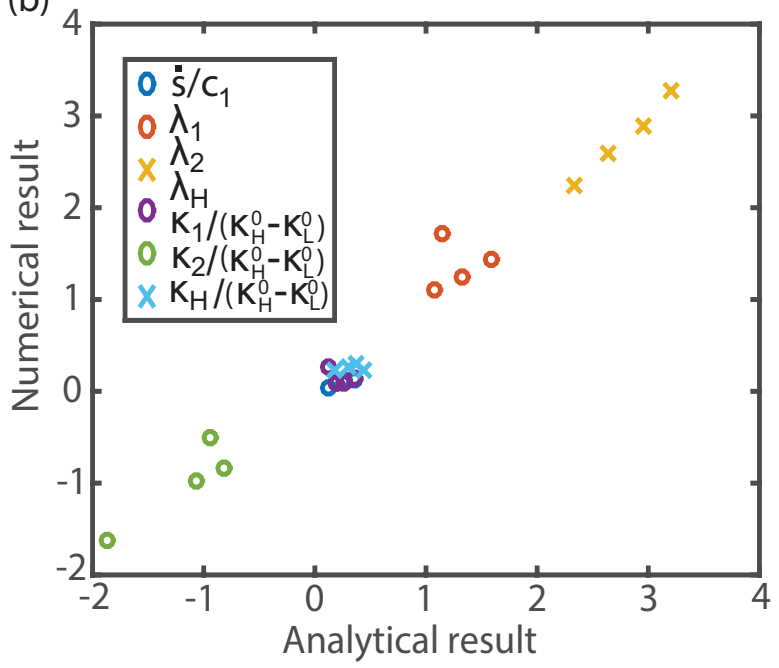

Figure 11: (a) Comparison of results of Riemann problem with static phase boundary from numerical integration and analytical solution using jump conditions. We have used $B_{1}=10, B_{2}=7.6, B_{3}=10$ and $B_{1}=10, B_{2}=3, B_{3}=10$. (b) Comparison of numerical results with analytical solution of impact problems for $B_{1}=10, B_{2}=3, B_{3}=10$ when $\dot{s}<c_{2}$. The analytical results are obtained from the jump conditions using phase boundary velocity from the numerical calculation.

from our calculations satisfies equation 30. However, we find that in these cases with $\dot{s}<c_{2}$, the dissipation rate is negative, $\dot{s} f_{\text {driving }}<0$. Starting from eqn. (28) we see that negative dissipation rates occur whenever

$$
\dot{s} / c_{1}<\sqrt{\left(1-f_{\text {threshold }}\right)\left(c_{2} / c_{1}\right)^{2}-f_{\text {threshold }}} .
$$

On the $\dot{s}-c_{2}$ plane (see figure 12) the region represented by the above inequality overlaps with the region corresponding to $\dot{s}<c_{2}$. It seems that it is easier to see negative dissipation rates when we have another 'knob to turn' that is not available in an atomic chain with only extensional degrees of freedom. That 'knob' 
is the wave speed $c_{2}$. We can pick $c_{2}$ and initial/boundary conditions of a Riemann/impact problem in such a way as we sit in the region corresponding to negative dissipation rates in the $\dot{s}-c_{2}$ plane. If this idea is correct we might expect similar phenomena in 2D lattice simulations of phase-transforming materials in which both P- and S-waves are present. However, such simulations are outside the scope of this paper.

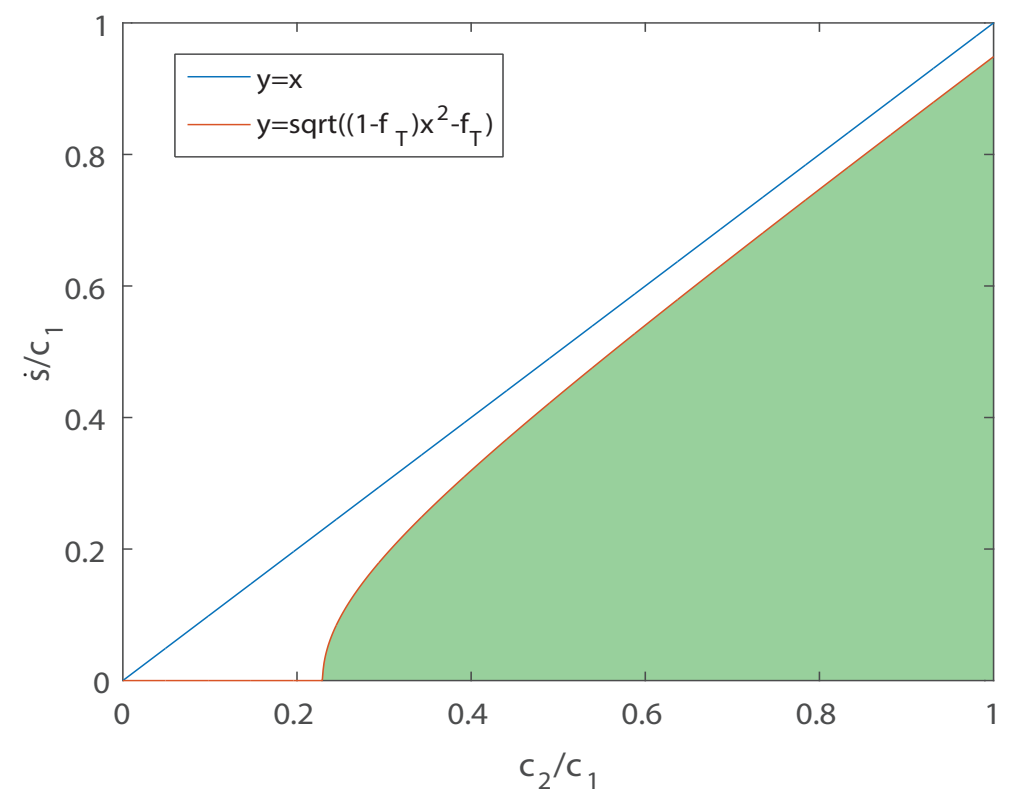

Figure 12: Region corresponding to negative dissipation rates in the $\dot{s}-c_{2}$ plane is colored green. In the legend $f_{T}$ stands for $f_{\text {threshold }}, x$ stands for $c_{2} / c_{1}$ and $y$ stands for $\dot{s} / c_{1}$.

What are the implications of a negative dissipation rate? Naively, it means that at time $t$ the chain has more energy than what it had at $t=0$ plus what was injected into it at the boundary. This is unphysical and cannot be true. The reason behind this anomaly is that in calculating the driving force using data from our numerical calculations we have used an isothermal expression derived from continuum theory that only uses the average values of the stretches, velocities, etc., and neglects the oscillations of the masses and springs. In fact, it has been suggested in the the literature that simulations of phase transitions using mass-spring chains need to be done with new variables describing level of fluctuations around average stretches, velocities, etc., as well as the rate of energy fluxes into various scales and that into irreversible dissipation by elastic radiation [20, 25, 5, 27]. Dayal and Bhattacharya [11] have considered oscillations around average stretches and velocities in a peridynamic setting and found that they are of the same magnitude ahead and behind a phase boundary, leading to a zero driving force. This is not the case with oscillations in our calculations so we expect non-zero driving forces, but negative dissipation rates are still puzzling. To ensure that our results of negative dissipation rates are not spurious we performed an energy budget on our chain. We calculated the energy input over time $t=J \Delta t$ as $E_{i n}(t)=\sum_{i=1}^{J} F_{N+\frac{1}{2}}^{i} v_{0} \Delta t+\sum_{i=1}^{J} M_{N+\frac{1}{2}}^{i} w_{0} \Delta t$, and the energy of the chain as $E_{\text {chain }}(t)=\sum_{p=1}^{N} \frac{1}{2} \dot{u}_{p}^{2}(t)+\sum_{p=1}^{N} \dot{\theta}_{p}^{2}(t)+\phi\left(u_{1}(t), u_{2}(t), \ldots, u_{N+1}(t), \theta_{1}(t), \theta_{2}(t), \ldots \theta_{N+1}(t)\right)$. We have 
plotted the difference between input energy and the total energy of the chain $E_{i n}-E_{c h a i n}$ as a function of $t=J \Delta t$ in figure 14 for three different impact problems with a single phase boundary. In all these impact problems the initial conditions were such as the potential energy of the springs and kinetic energy of the masses was zero at $t=0$. For the top two curves (red and blue) the dissipation rate at the phase boundary is positive, while for the bottom (yellow) curve it is negative. In all cases the difference $E_{\text {in }}-E_{\text {chain }}$ is positive at all times. This is as it should be since our numerical integration scheme is imperfect and it results in some numerical dissipation. Notice from these curves that after an initial transient the difference $E_{\text {in }}-E_{\text {chain }}$ tends to a constant. In other words, the rate of energy input is equal to the rate of energy (kinetic + potential) storage in the chain after an initial transient. These observations suggest that we cannot neglect the jump in the magnitude of energy stored in the oscillations, or, loosely speaking, the jump in temperature, across a moving phase boundary.
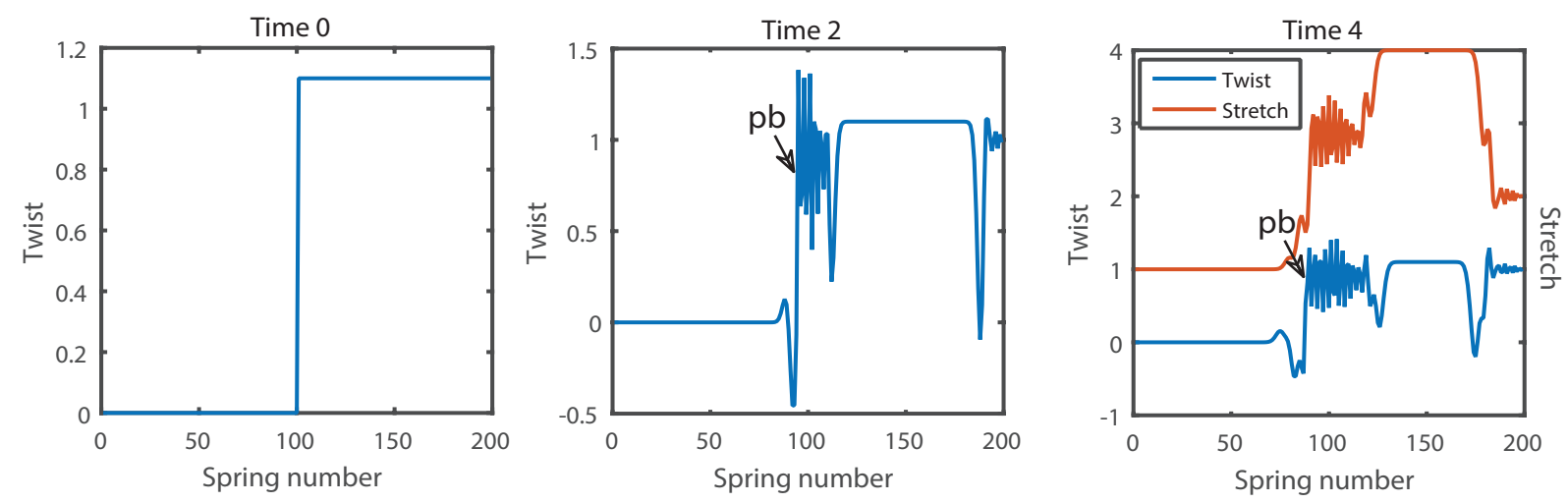

Figure 13: An example of an Riemann problem in which $\dot{s}<c_{2}$. Fives waves are present, four sonic waves at speeds $c_{1}$ and $c_{2}$ propagating in opposite directions from the middle, and the phase boundary $\dot{s}<c_{2}$. The panels show snapshots of the twist in the chain as function of spring number at six different times. The bottom two panels also show snapshots of the stretch.

\subsection{Impact problem for adiabatic process}

To re-examine the negative dissipation rate cases, we introduce a temperature in our analysis defined locally through

$$
k \Theta_{j}(t)=K E_{j}(t)+P E_{j+\frac{1}{2}}(t)-E_{j}^{c o n t}=\frac{1}{2} m_{j} \dot{u}_{j}^{2}(t)+\frac{1}{2} I_{j} \dot{\theta}_{j}^{2}(t)+\phi_{1}\left(x_{j}(t), y_{j}(t)\right)-E_{j}^{c o n t} .
$$

Here, $K E_{j}(t)$ is the kinetic energy of the $j^{t h}$ mass (accounting for both translational and rotational contributions) at time $t, P E_{j+\frac{1}{2}}(t)$ is the potential energy of the spring between mass $j$ and mass $j+1$ at time $t$ and $E_{j}^{\text {cont }}$ is the sum of kinetic energy at mass $j$ and potential energy in spring $j+\frac{1}{2}$ computed using the average values of the velocities and strains that satisfy the continuum jump conditions,

$$
E_{j}^{\text {cont }}=\frac{1}{2} m_{j}\left\langle\dot{u}_{j}\right\rangle^{2}+\frac{1}{2} I_{j}\left\langle\dot{\theta}_{j}\right\rangle^{2}+\phi_{1}\left(\left\langle x_{j}\right\rangle,\left\langle y_{j}\right\rangle\right)
$$


(a)

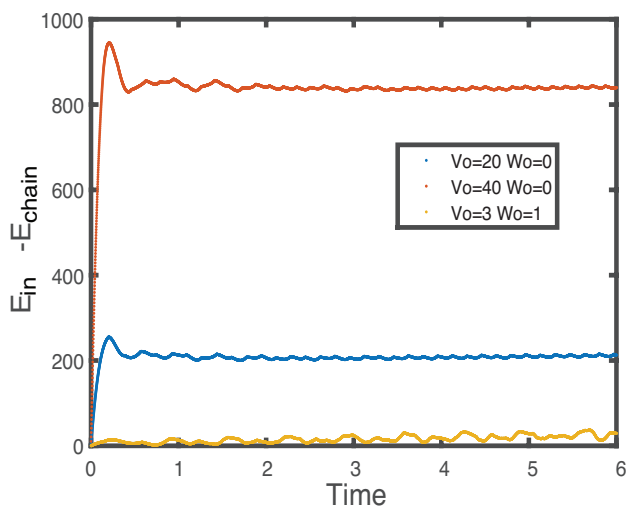

(b)

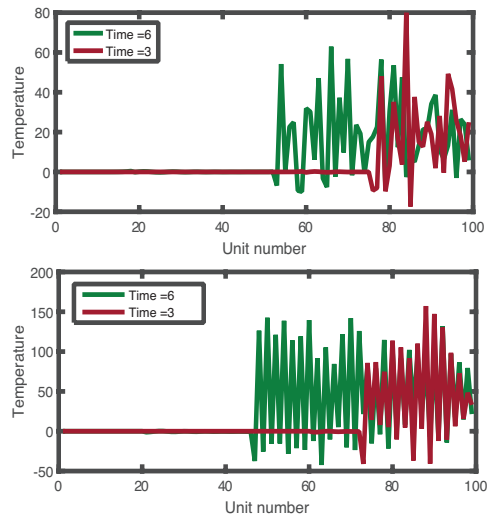

(c)

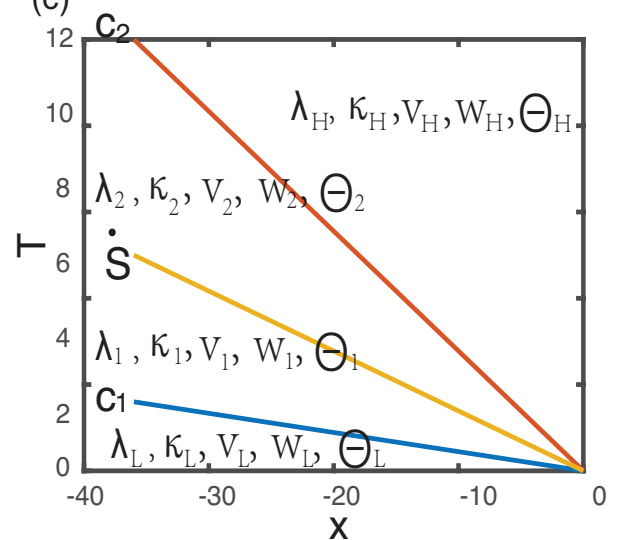

Figure 14: (a)Difference between input energy and PE+KE of the whole chain for three different impact problems on a chain with $B_{1}=10, B_{2}=3, B_{3}=10$. The red and blue curves involve a phase boundary in which dissipation rate is positive and the yellow curve involves a phase boundary in which dissipation rate is negative. (b) Snapshots of the local temperature $\Theta_{j}$ in the chain as a function of mass number $j$ for two different boundary conditions. The top panel shows snapshots at two different times (red and green) for boundary condition $v_{0}=10, w_{0}=0$, and the bottom panel shows snapshots for boundary condition $v_{0}=7, w_{0}=0$. Note that the temperature jumps only once (across the phase boundary) even though there are three propagating discontinuities in the impact problem on the chain. (c) The new x-t plane when temperature is considered in an adiabatic impact problem on a thermoelastic bar of the Mie-Gruneisen type.

where $\langle z\rangle$ is the temporal average of $z$ over a time corresponding to few periods. $k$ is proportional to a specific heat in the continuum theory and we set it equal to 1 here. So, our definition of the local temperature captures the 'thermal' portion of the energy of the masses and springs in the chain that is neglected in a purely mechanical continuum theory. We could also have defined two different temperatures [16] corresponding to rotational and extensional degrees of freedom, respectively, but we do not do so here because the temperature defined above has the property that it satisfies jump conditions expected of a Mie-Gruniesen type thermoelastic material (see the appendix for a short review of these materials). This is discussed in the following.

Since our numerical method is such as there is no leakage of energy (thermal + mechanical) from our chain we expect that the phase boundary propagation is adiabatic. To confirm if this is the case we plot the local temperature $\Theta_{j}$ in our chain for an impact problem as a function of mass number $j$ in figure 14(b). We find that $\Theta$ does not jump across sonic waves which move at speeds $c_{1}$ and $c_{2}$, but it jumps across a phase boundary. Furthermore, $\Theta$ oscillates around a constant value behind a phase boundary. Thus, if we take a temporal average value of $\Theta$, then our $\mathrm{x}$-t plane is very similar to the $\mathrm{x}$-t plane for an adiabatic impact problem of a trilinear Mie-Gruneisen thermoelastic solid in which the Gruneisen parameter is zero [1]. To make this analogy more concrete we write the jump conditions at each discontinuity in an impact problem on a bar in which both stretching and twisting are allowed and the local temperature can suffer 
jumps. We assume that the Gruneisen parameter is zero and that the continuum problem is adiabatic. The jump conditions are:

$$
\begin{aligned}
& \text { Across }-c_{1}\left\{\begin{array}{l}
-c_{1}\left(\lambda_{1}-\lambda_{L}\right)+v_{1}-v_{L}=0, \\
-c_{1}\left(\kappa_{1}-\kappa_{L}\right)+w_{1}-w_{L}=0, \\
-c_{1}\left(v_{1}-v_{L}\right)+S\left(\lambda_{1}-\lambda_{L}\right)+g\left(\kappa_{1}-\kappa_{L}\right)=0, \\
\Theta_{1}-\Theta_{L}=0,
\end{array}\right. \\
& \text { Across }-c_{2}\left\{\begin{array}{l}
-c_{2}\left(\lambda_{H}-\lambda_{2}\right)+v_{H}-v_{2}=0, \\
-c_{2}\left(\kappa_{H}-\kappa_{2}\right)+w_{H}-w_{2}=0, \\
-c_{2}\left(v_{H}-v_{2}\right)+S\left(\lambda_{H}-\lambda_{2}\right)+g\left(\kappa_{H}-\kappa_{2}\right)=0, \\
\Theta_{H}-\Theta_{2}=0,
\end{array}\right. \\
& \text { Across } \dot{s}\left\{\begin{array}{l}
\dot{s}\left(\lambda_{2}-\lambda_{1}\right)+v_{2}-v_{1}=0, \\
\dot{s}\left(\kappa_{2}-\kappa_{1}\right)+w_{2}-w_{1}=0, \\
\dot{s}\left(v_{2}-v_{1}\right)+S\left(\lambda_{2}-\lambda_{1}\right)+g\left(\kappa_{2}-\kappa_{1}\right)=0, \\
\dot{s}\left(w_{2}-w_{1}\right)+g\left(\lambda_{2}-\lambda_{1}-\lambda_{H}^{0}+\lambda_{L}^{0}\right)+C\left(\kappa_{2}-\kappa_{1}-\kappa_{H}^{0}+\kappa_{L}^{0}\right)=0, \\
\rho C_{p}\left(\Theta_{2}-\Theta_{1}\right)=\frac{T_{2}+T_{1}}{2}\left(\lambda_{0}^{H}-\lambda_{0}^{L}\right)+\frac{M_{2}+M_{1}}{2}\left(\kappa_{0}^{H}-\kappa_{0}^{L}\right)
\end{array}\right.
\end{aligned}
$$

The appropriate expression for the driving force across a phase boundary for such a material (with zero latent heat) is

$$
f_{\text {driving }}=-E \log \left(1+\frac{[|\Theta|]}{\Theta_{-}}\right),
$$

where we treat $\Theta_{-}$and $E$ as constants. In the continuum impact problems solved in [1] $\Theta_{-}$is set equal to the transformation temperature and $E$ is related to the specific heat. We find that our numerical simulations of the mass-spring chain with $B_{1}=10, B_{2}=3, B_{3}=10$ satisfy these jump conditions if we take $\rho C_{p} \approx 0.07463$. We also find that $[|\Theta|]<0$ irrespective of whether $\dot{s}<c_{2}$ or $\dot{s} \geq c_{2}$. Thus, the dissipation rate $f_{\text {driving }} \dot{s}$ computed using (34) is always non-negative for the phase boundaries in our chain. The kinetic relation using this new driving force is plotted in figure 15. While we arrived at this kinetic relation through a bistable chain having both extensional and rotational degrees of freedom, this is not necessary. This can be seen from the red curve in figure 15 which is the kinetic relation of a bistable chain with only extensional degrees of freedom. Remarkably, the curves are identical except for the value of the constant $\Theta_{-}$. 


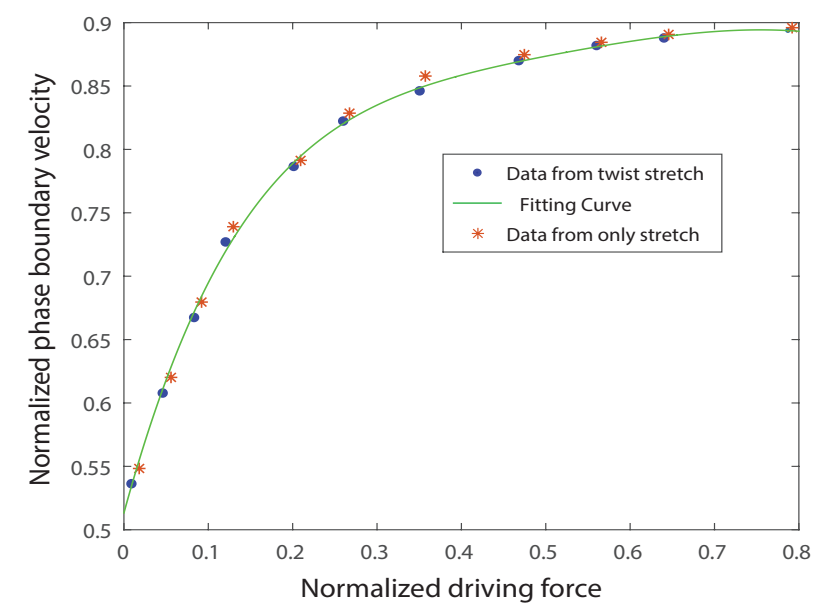

Figure 15: The new kinetic relation defined by 34 which accounts for the jump in temperature across a phase boundary. The "." data are results from calculation with twist stretch coupling and the "*" data are results from calculation with only extensional degree of freedom. We use a fitting curve $f(x)=a b x^{b-1} \exp \left(-a x^{b}\right)$ where $a=1.181, b=1.139, E=1$. $\Theta_{-}=1000$ for twist stretch coupling data and $\Theta_{-}=622$ with only extensional degrees of freedom.

\section{Application to artificial muscle yarns}

We now want to demonstrate a practical application where methods developed in this paper could be useful. In [17] carbon nanotube yarns are twisted, braided and then filled with wax to produce filaments that are capable of actuation. Actuation is realized by heating the filaments to cause partial melting (a phase transition) of the wax which does not flow out of the yarn due to favorable adhesive interactions with the nanotubes over very large surface areas. The mechanism behind the actuation is the change in pitch of the helical yarns due to softening of the wax. Thus, twist and stretch are coupled in these yarns and it's a phase transition that enables its reversible operation over many cycles. Our goal here is to model one aspect of the yarn's behavior that has been deduced in the experiments of [17]. In these experiments different types of yarn are loaded with a constant tensile force. Then they are heated by an electrical current for several tens of milliseconds. This causes the yarn to stretch, so that useful work is done by the applied tension. The tensile actuation strain is measured in the experiment and plotted as a function of time as the yarn cools in figure 3 of [17]. The end of the yarn also rotates at high angular velocities due to the coupling of twist and stretch. Two types of responses are seen for the tensile actuation strain as a function of time - (a) a monotonically decaying strain, and (b) a decaying oscillatory strain. We provide a possible explanation of these two behaviors.

Consider first the monotonically decaying strain. At $t=0$ the yarn is at temperature $T=T_{1}$ due to the electrical heating. Here, $T_{1}>T_{\text {air }}$ where $T_{\text {air }}$ is the ambient temperature. The change in length of the filament $\Delta L$ is proportional to $\Delta T=T-T_{\text {air }}$ where $T(t)$ is the current temperature of the yarn. Due to convective and radiative heat transfer to the environment one can write an equation for $\frac{d T}{d t}$ at a rudimentary 
level [29]:

$$
-\frac{d T}{d t}=a\left(T-T_{a i r}\right)+b\left(T^{4}-T_{a i r}^{4}\right),
$$

where $a$ is a constant that depends on the convective heat transfer coefficient, density, specific heat and geometry of the yarn, etc., and $b$ is another constant that depends on a radiative heat transfer coefficient and other yarn parameters. This equation can be integrated with initial condition $T(t=0)=T_{1}$ to get a monotonically decaying $T$. This will lead to a monotonically decaying strain $\Delta L$ as a function of $t$ because $\Delta L$ is assumed proportional to $\Delta T$ in [17]. If we neglect the radiative terms then the solution of the differential equation above is simply $T(t)=T_{a i r}+\left(T_{1}-T_{a i r}\right) \exp (-a t)$. This rudimentary analysis cannot explain the observed oscillatory decay of the strain in some other yarn samples. A possible explanation for this phenomenon combines dynamics of the yarn with the above heat transfer analysis. We model the yarn with our mass-spring chain coupling twist and stretch. Initially, all the springs are at the bottom of the low strain well and the masses are stationary. Then at $t=0$ we suddenly apply a constant force $F$ and constant moment $M=0$ on mass $N+1$ while mass 1 is fixed. We plot the displacement $u_{N+1}(t)=L \epsilon(t)=\Delta L$ as a function of time in figure 16. Due to the sonic waves and phase boundary going back and forth in the chain the displacement oscillates. In our simulation we cannot account for the heat transfer to the environment or the change in $L$ due to changes in temperature, but from the above analysis we know that $L(t)$ decays as a function of time, so $\Delta L$ will decay in an oscillatory fashion because $\epsilon(t)$ oscillates. We show this in figure $16(\mathrm{~b}, \mathrm{c})$.
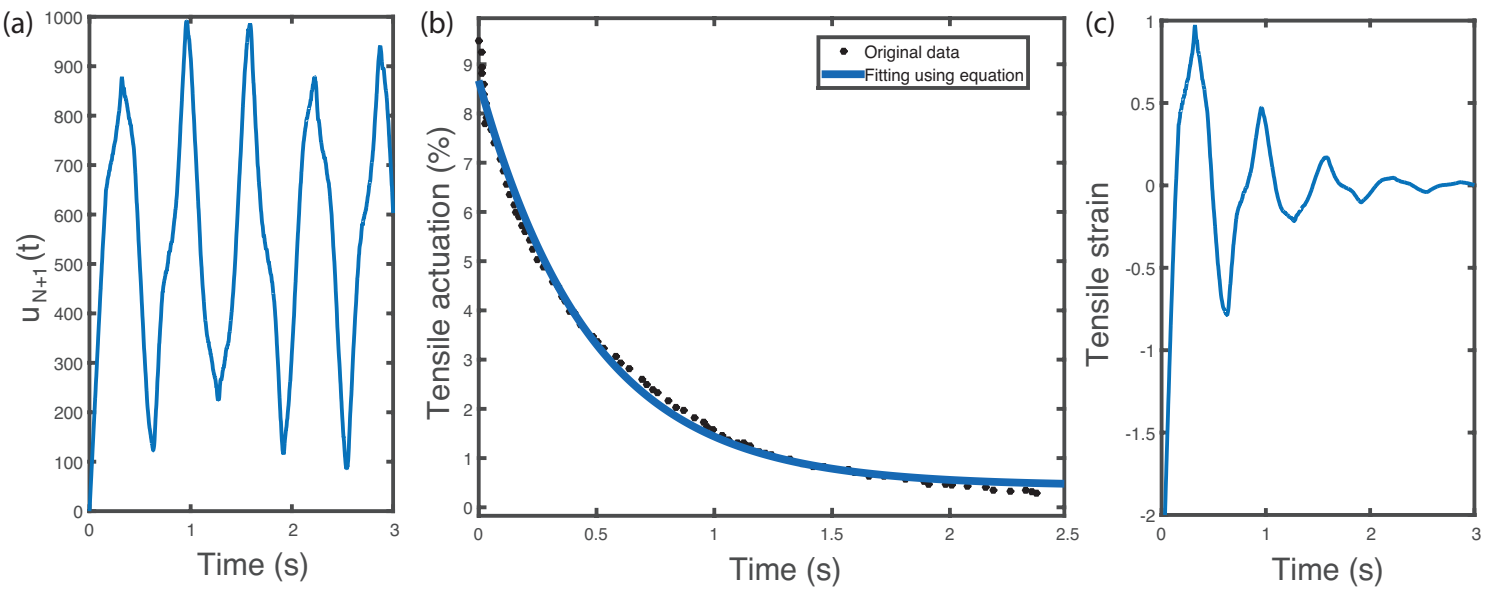

Figure 16: Simulation of the experiment in [17] using our mass-spring chain. We apply a constant force and moment at the end of the chain as our boundary conditions. We simulate to the point in time when the phase boundary has hit the left end and bounced back. We recover the vibration of the tensile actuation strain with our numerical result coupled with a decaying function described in equation 35. We have plotted the following. (a) Total length of chain from numerical simulation. (b) Experimental data in [17] for tensile actuation strain fitted using equation 35. (c) Oscillatory actuation strain of the chain from numerical result coupled with a decaying envelope qualitatively captures what is seen in other experiments in [17]. 


\section{Conclusions}

In this paper we have studied the dynamics of a mass-spring chain with extensional and rotational degrees of freedom at each mass. The energy landscape of the springs has multiple wells, as would be the case for a rod capable of phase transitions. We have shown that the solutions of continuum Riemann and impact problems in rods capable of twist-stretch phase transitions are analogous to the those in the numerical solutions of our chains. In particular, we have observed discontinuities across which the twist and stretch can jump. Discontinuities of this type had been used to model impact of elasto-plastic bars and nano tubes $[8,28,19]$, but we are not aware of an attempt to use them in the study of phase transitions, except [3]. Our mass-spring chain has furnished a kinetic relation for phase boundaries across which the twist and stretch can jump. This kinetic relation has a form that is familiar from earlier studies involving chains with only extensional degrees of freedom. However, for some combinations of parameters characterizing the energy landscape of the springs we find propagating phase boundaries for which the rate of dissipation, as calculated using isothermal expressions for the driving force, is negative. This is a surprising result which suggests that we cannot neglect the energy stored in the oscillations of the masses in the interpretation of the dynamics of mass-spring chains. In particular, we must incorporate temperature into the numerical solutions of these chains. We have made an attempt to do so by defining a temperature that is proportional to the energy in the vibrations of the masses and springs that a purely mechanical continuum theory neglects. A temperature defined in this way jumps only across a phase boundary and not a sonic wave. This is exactly what happens in a Mie-Gruneisen type material with the Gruneisen parameter equal to zero. We have used this insight to extract a new kinetic relation that does not suffer from the anomaly of giving negative dissipation rates at a moving phase boundary. Does this mean that our earlier kinetic relation that was based on isothermal expressions of the driving force is useless? We do not think so for the following reason. In [36] we implemented a Langevin dynamics algorithm in which it was possible to simulate a mass-spring chain immersed in a constant temperature bath. We showed that the local temperature near a phase boundary was high but it soon decayed to the temperature of the bath due to heat transfer. We found that the kinetic relation derived using isothermal expressions was not affected much by the bath temperature, especially for large phase boundary velocities. Hence, we expect that kinetic relation summarized in equation (30) will be a good approximation at the near sonic limit. At the end of the paper we use our chain to shed some light on experiments involving temperature sensitive yarns that couple twist and stretch to perform useful work. We have shown that it is important to consider the dynamics of the yarn, especially reflection of sonic waves and/or phase boundaries at the ends of the yarn, in order to explain the oscillatory decay of the actuation strain due to cooling of the yarn at fixed tension. However, we must point out that there are several limitations of our model, including (a) we have not included a spinodal region in the constitutive law, (b) we have not included a heat bath, (c) we have not included bending deformations. All three are 
important and we plan to include them in future work.

\section{Acknowledgements}

We acknowledge support from NSF through grant number NSF CMMI 1066787 and through the Nano/Bio Interface Center at the University of Pennsylvania through grant number NSF DMR08-32802.

\section{Appendix}

Here we give a short summary of Mie-Gruneisen type thermoelastic material with only a translational degree of freedom. A detailed description of phase transitions in this type of material can be found in [1]. The constitutive relations for the stress and entropy of a two-phase Mie-Grueisen material in one dimension are given by:

$$
\begin{gathered}
T= \begin{cases}S \lambda-\rho c \beta\left(\theta-\theta_{T}\right), & \text { for the ' } \mathrm{L} \text { ' phase, } \\
S\left(\lambda+\gamma_{T}\right)-\rho c \beta\left(\theta-\theta_{T}\right), & \text { for the 'H' phase, }\end{cases} \\
\eta= \begin{cases}\beta c \lambda+c\left(1+\log \left(\theta / \theta_{T}\right)\right), & \text { for the ' } \mathrm{L} \text { ' phase, } \\
\beta c\left(\lambda+\gamma_{T}\right)+c\left(1+\log \left(\theta / \theta_{T}\right)\right)-\lambda_{T} / \theta_{T}, & \text { for the ' } \mathrm{H} \text { ' phase, }\end{cases}
\end{gathered}
$$

where $S$ is the stretching modulus, $\lambda$ is stretch, $\rho$ is density, $\theta$ is temperature, $c$ is specific heat, $\beta$ is Gruneisen parameter and $\gamma_{T}, \lambda_{T}$ and $\theta_{T}$ are constants. For a propagating discontinuity in a bar made of such a material the following jump conditions must be satisfied under adiabatic conditions:

$$
\begin{aligned}
{[|v|]+\dot{s}[|\lambda|] } & =0, \\
{[|T|]+\rho \dot{s}[|v|] } & =0, \\
(\rho[|c \theta|]-\langle T\rangle[|\lambda|]) \dot{s} & =0,
\end{aligned}
$$

where $v$ is the particle velocity. The driving force at such a discontinuity is given by:

$$
f_{\text {driving }}=-\rho\langle\theta\rangle[|\eta|] .
$$

We can specialize this to a phase boundary by substituting the appropriate constitutive relations for $\eta$. If we assume $\beta=0$ we get for a phase boundary

$$
f_{\text {driving }}=\left(\lambda_{T} / \theta_{T}+c \log \left(\theta^{+} / \theta^{-}\right)\right)
$$

If $\lambda_{T}$ (the latent heat) is also zero (since the bottom of the two wells are at the same height) then we are left with $f_{\text {driving }}=c \log \left(\theta^{+} / \theta^{-}\right)$which is what we have used as the driving force in the text. 


\section{Reference}

[1] Abeyaratne, R., Knowles, J. K., 2006. Evolution of phase transitions: a continuum theory. Cambridge University Press.

[2] Abeyaratne, R., Vedantam, S., 2003. A lattice-based model of the kinetics of twin boundary motion. Journal of the Mechanics and Physics of Solids 51 (9), 1675-1700.

[3] Abeyaratne, R. C., 1981. Discontinuous deformation gradients in the finite twisting of an incompressible elastic tube. Journal of Elasticity 11 (1), 43-80.

[4] Agrawal, V., Dayal, K., 2015. A dynamic phase-field model for structural transformations and twinning: Regularized interfaces with transparent prescription of complex kinetics and nucleation. part i: Formulation and one-dimensional characterization. Journal of the Mechanics and Physics of Solids 85, 270-290.

[5] Balk, A. M., Cherkaev, A. V., Slepyan, L. I., 2001. Dynamics of chains with non-monotone stress-strain relations. i. model and numerical experiments. Journal of the Mechanics and Physics of Solids 49 (1), 131-148.

[6] Benichou, I., Givli, S., 2011. The hidden ingenuity in titin structure. Applied Physics Letters 98 (9), 091904.

[7] Benichou, I., Givli, S., 2013. Structures undergoing discrete phase transformation. Journal of the Mechanics and Physics of Solids 61 (1), 94-113.

[8] Clifton, R., 1966. An analysis of combined longitudinal and torsional plastic waves in a thin-walled tube. Tech. rep., DTIC Document.

[9] Cohen, T., Givli, S., 2014. Dynamics of a discrete chain of bi-stable elements: A biomimetic shock absorbing mechanism. Journal of the Mechanics and Physics of Solids 64, 426-439.

[10] Dayal, K., Bhattacharya, K., 2006. Kinetics of phase transformations in the peridynamic formulation of continuum mechanics. Journal of the Mechanics and Physics of Solids 54 (9), 1811-1842.

[11] Dayal, K., Bhattacharya, K., 2007. A real-space non-local phase-field model of ferroelectric domain patterns in complex geometries. Acta materialia 55 (6), 1907-1917.

[12] Falk, W., James, R. D., 2006. Elasticity theory for self-assembled protein lattices with application to the martensitic phase transition in bacteriophage t4 tail sheath. Physical Review E 73 (1), 011917.

[13] Goodstein, D. L., 2014. States of matter. Courier Corporation.

[14] Kittel, C., 2005. Introduction to solid state physics. Wiley.

[15] Koehler, T., Bishop, A., Krumhansl, J., Schrieffer, J., 1975. Molecular dynamics simulation of a model for (onedimensional) structural phase transitions. Solid State Communications 17 (12), 1515-1519.

[16] Langer, J. S., Egami, T., 2012. Glass dynamics at high strain rates. Physical Review E 86 (1), 011502.

[17] Lima, M. D., Li, N., De Andrade, M. J., Fang, S., Oh, J., Spinks, G. M., Kozlov, M. E., Haines, C. S., Suh, D., Foroughi, J., et al., 2012. Electrically, chemically, and photonically powered torsional and tensile actuation of hybrid carbon nanotube yarn muscles. Science 338 (6109), 928-932.

[18] Nadkarni, N., Daraio, C., Kochmann, D. M., 2014. Dynamics of periodic mechanical structures containing bistable elastic elements: From elastic to solitary wave propagation. Physical Review E 90 (2), 023204.

[19] Ng, K., Sun, Q., 2006. Stress-induced phase transformation and detwinning in niti polycrystalline shape memory alloy tubes. Mechanics of Materials 38 (1), 41-56.

[20] Ngan, S.-C., Truskinovsky, L., 1998. Thermal trapping and kinetics of martensitic phase boundaries. Journal of the Mechanics and Physics of Solids 47 (1), 141-172.

[21] Purohit, P. K., Bhattacharya, K., 2002. On beams made of a phase-transforming material. International journal of solids and structures 39 (13), 3907-3929.

[22] Purohit, P. K., Bhattacharya, K., 2003. Dynamics of strings made of phase-transforming materials. Journal of the Mechanics and Physics of Solids 51 (3), 393-424.

[23] Raj, R., Purohit, P. K., 2010. Moving interfaces in rod-like macromolecules. EPL (Europhysics Letters) 91 (2), 28003. 
[24] Raj, R., Purohit, P. K., 2011. Phase boundaries as agents of structural change in macromolecules. Journal of the Mechanics and Physics of Solids 59 (10), 2044-2069.

[25] Rosakis, P., Knowles, J. K., 1997. Unstable kinetic relations and the dynamics of solid-solid phase transitions. Journal of the Mechanics and Physics of Solids 45 (11), 2055-2081.

[26] Schwetlick, H., Zimmer, J., 2012. Kinetic relations for a lattice model of phase transitions. Archive for Rational Mechanics and Analysis 206 (2), 707-724.

[27] Slepyan, L., Troyankina, L., 1984. Fracture wave in a chain structure. Journal of Applied Mechanics and Technical Physics $25(6), 921-927$.

[28] Sun, Q.-P., Li, Z.-Q., 2002. Phase transformation in superelastic niti polycrystalline micro-tubes under tension and torsion--from localization to homogeneous deformation. International Journal of Solids and Structures 39 (13), $3797-3809$.

[29] Suryanarayana, N. V., 1995. Engineering heat transfer. West Publishing.

[30] Tam, B. K., Shin, J. H., Pfeiffer, E., Matsudaira, P., Mahadevan, L., 2009. Calcium regulation of an actin spring. Biophysical journal 97 (4), 1125-1129.

[31] Tan, L., Acharya, A., Dayal, K., 2013. Coarse variables of autonomous ode systems and their evolution. Computer Methods in Applied Mechanics and Engineering 253, 199-218.

[32] Truskinovsky, L., Vainchtein, A., 2005. Kinetics of martensitic phase transitions: lattice model. SIAM Journal on Applied Mathematics 66 (2), 533-553.

[33] Vainchtein, A., Kevrekidis, P. G., 2012. Dynamics of phase transitions in a piecewise linear diatomic chain. Journal of nonlinear science 22 (1), 107-134.

[34] van Mameren, J., Gross, P., Farge, G., Hooijman, P., Modesti, M., Falkenberg, M., Wuite, G. J., Peterman, E. J., 2009. Unraveling the structure of dna during overstretching by using multicolor, single-molecule fluorescence imaging. Proceedings of the National Academy of Sciences 106 (43), 18231-18236.

[35] Zhang, X., Chen, H., Le, S., Rouzina, I., Doyle, P. S., Yan, J., 2013. Revealing the competition between peeled ssdna, melting bubbles, and s-dna during dna overstretching by single-molecule calorimetry. Proceedings of the National Academy of Sciences 110 (10), 3865-3870.

[36] Zhao, Q., Purohit, P. K., 2014. Extracting a kinetic relation from the dynamics of a bistable chain. Modelling and Simulation in Materials Science and Engineering 22 (4), 045004.

[37] Zhao, Q., Purohit, P. K., 2015. Phase boundaries with discontinuous stretch and twist in dna. submitted to International Journal of Solids and Structures. 